\title{
The gall midges (Diptera, Cecidomyiidae) from three restingas of Rio de Janeiro State, Brazil
}

\author{
Valéria Cid Maia ${ }^{1}$
}

\begin{abstract}
One hundred and eight species of Cecidomyiinae (Cecidomyiidae) were found in association with 53 species of plant distributed among 42 genera and 32 families at restingas of Barra de Maricá, Itaipuaçu and Carapebus. Ninety four gall midge species were cecidogenous, four predaceous, five inquilinous of galls and five were free living. Galling species were associated with 47 plant species belonging to 36 genera and 28 families. The majority of the galls occurred on the leaves $(\mathrm{N}=63)$; 13 on buds; nine on inflorescence, closed flower or flower peduncle; three on fruits and one on tendril. Myrtaceae were the richest plant family in number of galls followed by Burseraceae, Nyctaginaceae, Sapotaceae, Erythroxylaceae, Malpighiaceae and Solanaceae. New records of host plants and localities were recorded. Seventy nine Cecidomyiinae species were found at Restinga of Barra de Maricá, 64 at Carapebus and 41 at Itaipuaçu. Sorensen's index revealed that the restingas of Barra de Maricá and Itaipuaçu are more similar in Cecidomyiinae fauna, confirming a positive relation between geographical proximity and fauna similarity.

KEY WORDS. Cecidomyiidae, Diptera, gall, restinga
\end{abstract}

The restinga or coastal shrub zone is a very complex environment whose diversity is exceeded only by rain forests. Their flora has been originated from the Atlantic Forest and the plant families best represented in these areas are Leguminosae, Rubiaceae, Orchidaceae, Myrtaceae, Poaceae, Bromeliaceae and Compositae. Among them, Myrtaceae contributes significantly to its flora characterisation, being Eugenia Linnaeus the biggest genus in number of species (RIZZINI 1979).

Cecidomyiidae are the most common gall makers throughout the world. They comprise about 5000 known species, but only 500 have been recorded in the Neotropical region, reflecting the scarcity of taxonomic studies in this zoogeographical region.

Cecidomyiidae are divided into three subfamilies: Lestremiinae, Porricondylinae and Cecidomyiinae. The first two include mycophagous and free-living phytophagous species. The last includes all gall makers, as well as predators, inquilines, free-living phytophagous and mycophagous species. As the Cecidomyiinae comprise species of economic interest, it is much better known than the other subfamilies.

Besides the gall makers, other organisms are commonly found inside the gall, living as inquilines, predators or parasitoids. The parasitoids are considered to be the most important natural enemies of gall midges. MONTEIRO et al. (1993) recorded 93 kinds of insect galls in the restingas of Barra de Maricá and Arraial do

1) Departamento de Entomologia Museu Nacional. Quinta da Boa Vista, São Cristóvão, 20940-040 Rio de Janeiro, Rio de Janeiro. E-mail: maiavcid@acd.ufrj.br 
Cabo (Rio de Janeiro State). About $75 \%$ of them appeared to be induced by Cecidomyiidae (Diptera). Parasitoids species were found in $85 \%$ of these galls. More specific data on parasitoids in restinga galls can be found in MAIA \& Monteiro (1999a) and MAiA (1999).

The present study surveyed three different restingas of Rio de Janeiro State: Barra de Maricá, Itaipuaçu and Carapebus, whose flora is relatively well known due to surveys developed mainly by ARAÚJO \& HENRIQUES (1984), SILVA \& SOMNER (1984), Silva \& OliVEIRA (1989) and ARAúJo et al. (1998). The first two restingas are geographically continuous and belong to the city of Maricá. The other one is about $170 \mathrm{~km}$ away from Maricá.

The Restinga of Barra de Maricá (hereafter RBM) has been a protected area since 1984. It spreads over about 150 ha. The Restinga of Carapebus (hereafter RC) is part of the "Parque Nacional de Jurubatiba", whose area is about 15000 ha, Carapebus comprising $40 \%$ of it. This is the only park that includes a restinga area. While RBM and RC are well preserved, the restinga of Itaipuaçu (hereafter RI) has been partially destroyed by human activities (mainly the building industry).

\section{MATERIAL AND METHODS}

The restingas of Barra de Maricá, Itaipuaçu and Carapebus were investigated over a period of 12 months: RBM from January to December, 1997; RI from November, 1997 to October, 1998 and RC from May, 1998 to April, 1999. Collections were made monthly along two pathways in RBM (Fig. 1), one of them adjacent to the Zacarias Beach (ZBP) and the other adjacent to the Maricá lagoon (MLP). Collections were made along the "Avenida Beira-Mar" (Beira-Mar Avenue) in RI (Fig. 1), and along two differents pathways in RC, one near Carapebus lagoon and the other along the access road to "Praia Grande" (PGP) (Fig. 1). The vegetation was examined at each 200 meter point along these pathways for 30 minutes per visit. The geographic coordinates of the first and the last points of collection in each road were obtained using the Global System Position 45 (GPS 45). They are: RBM, MLP

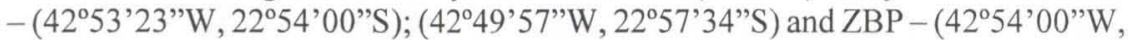

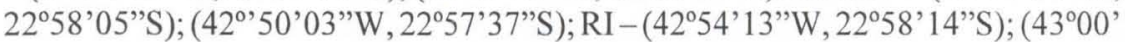

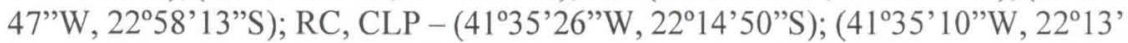

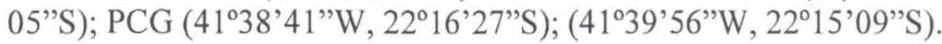

Samples of host plant, preferably with flowers and fruits, were pressed for preservation. They were later identified mainly by MsC Andrea Costa and Dr. Rui Alves (Departamento de Botânica, Museu Nacional, hereafter MNRJ). After having received a registration number, the dried plant specimens were incorporated into MNRJ herbarium. The system of plant classification is that proposed by CRONQUIST (1988) was followed, except for the Leguminosae, as suggested by the Herbarium curator.

Data on plant species distribution was mainly based on the literature: ARAÚJO et al. (in press) for Apocynaceae, Asclepiadaceae, Burseraceae, Cactaceae, Celastraceae, Erythroxylaceae, Euphorbiaceae, Fabaceae, Lauraceae (excepting Struthanthus maricensis), Malpighiaceae (excepting Tetrapteris phlomoides), Melastomataceae, Myrsinaceae, Eugenia uniflora, Myrciaria floribunda, Neomitranthes 


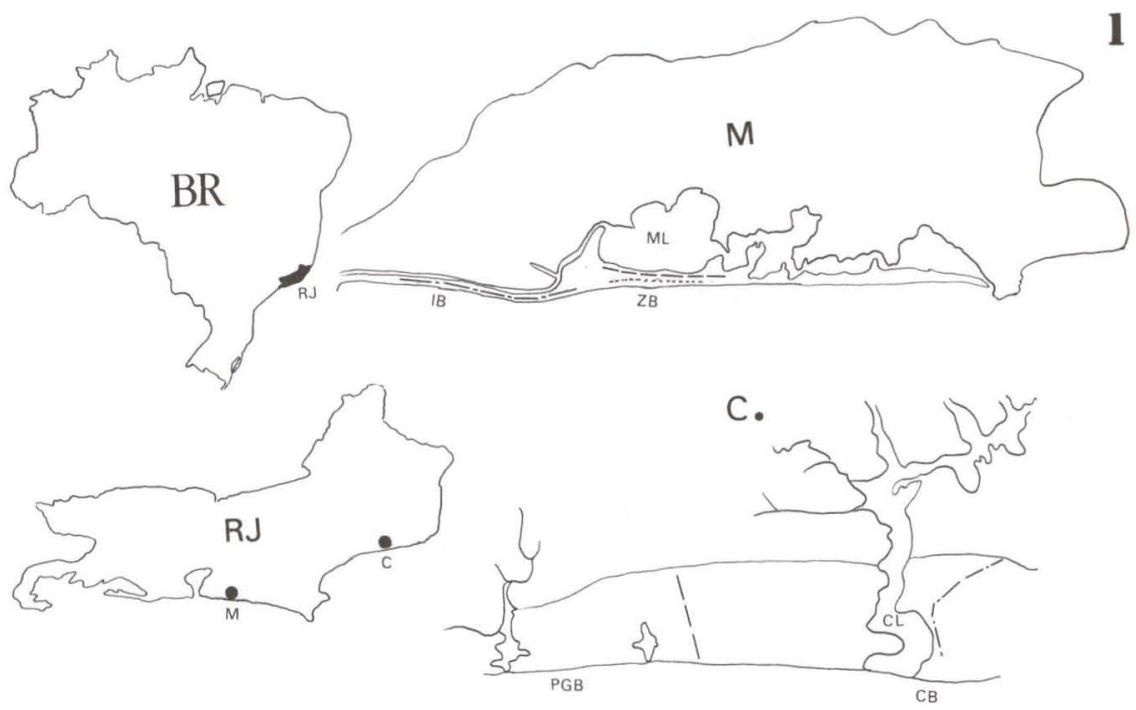

Fig. 1. Maps of Brazil, Rio de Janeiro State, Maricá city and Carapebus. (BR) Brazil, (C) Carapebus City, (CB) Carapebus Beach, (CL) Carapebus Lagoon, (M) Maricá City, (ML) Maricá Lagoon, (PGB) Praia Grande Beach, (RJ) Rio de Janeiro State, (ZB) Zacharias Beach, (MLP) Maricá Lagoon Pathway, (..... ZBP) Zacharias Beach Pathway, (...-...)Avenida Beira-mar (road).

obscura (Myrtaceae), Nyctaginaceae, Ochnaceae, Passifloraceae, Piperaceae, Rubiaceae, Sapindaceae, Manilkara subsericea (Sapotaceae), Smilacaceae, Solanaceae, Verbenaceae; PRANCE (1972) for Chrysobalanaceae; MARIz (1974) for Clusia lanceolata e Clusia fluminensis (Clusiaceae); MOREIRA \& RIzZINI (1997) for Struthanthus maricensis (Loranthaceae); BARROSO \& MARQUES (1997) for E. multiflora (Myrtaceae) and REITZ (1968) for Pouteria caimito var. laurifolia and P. venosa. Information about Mikania hoehnei (Asteraceae) and Jacquemontia holosericea (Convolvulaceae) was given by Dr. Roberto L. Esteves and Mariana S. Machado, respectively, both botanists at MNRJ. Data on Eugenia copacabanensis, Eugenia rotundifolia, Myrcia ovata (Myrtaceae) and Ximenia americana var. americana (Olacaceae) were obtained from the MNRJ Herbarium.

All studied galls were photographed and the negatives organised into an archive. Samples of dried galls are incorporated in the Diptera collection of MNRJ.

Larvae and pupae of the gall midges were obtained from the dissection of each kind of gall under a stereoscopic microscope. This procedure also enabled the determination of the gall dwellers' habits, whether inquilinous, predaceous, parasitoid or gall making.

The pupal exuviae and adults were obtained by keeping some samples of each kind of gall individually in plastic pots covered by a fine screening and layered at the bottom with damp cotton. Galls of the species whose larvae pupate in the soil were kept in pots containing a layer of restinga soil on the bottom. All pots were checked daily. 
Larvae, pupae, pupal exuviae and adults of Cecidomyiidae were preserved in $70 \%$ alcohol and they were later mounted on slides following the methodology of GAGNÉ (1994). The Cecidomyiidae genera were identified based mainly on the keys of GAGNÉ (1981, 1989, 1994).

The other arthropods - micro-Hymenoptera, Formicidae and Pseudoscorpiones - obtained from the galls were identified by Dr. John La Salle (International Institute of Entomology, Londres, England), Dr. Antônio Mayhé Nunes (Universidade Federal Rural do Rio de Janeiro, Brazil) and Dr. Mark Harvey (Western Australia Museum, Australia), respectively.

\section{RESULTS AND DISCUSSION}

One hundred and eight species of Cecidomyiidae were found in the three restingas studied. The most diverse one was $\mathrm{RBM}(\mathrm{N}=79)$, followed by $\mathrm{RC}(\mathrm{N}=$ $64)$ and $\mathrm{RI}(\mathrm{N}=41)$. The majority of them were gall makers $(\mathrm{N}=94)$, but some inquilinous, predaceous and free living species were found $(\mathrm{N}=5, \mathrm{~N}=4$ and $\mathrm{N}=$ 6 , respectively) (Tab. I).

Table I. Distribution of habts of gall midges in the restingas of Barra de Maricá (RBM), Itaipuaçu $(\mathrm{RI})$ and Carapebus (RC), Rio de Janeiro State.

\begin{tabular}{lcc}
\hline & \multicolumn{2}{c}{ Species } \\
\cline { 2 - 3 } Habits of gall midges & Number & Percentage (\%) \\
\cline { 2 - 3 } & 94 & 87,04 \\
Gall maker & 5 & 4,63 \\
Inquiline of galls & 4 & 3,70 \\
Predator in galls & 5 & 4,63 \\
Free living phytophagous & & \\
\hline
\end{tabular}

The gall midges were associated with 53 species of plants (42 genera and 32 families). Myrtaceae were the plant family with the greatest richness of galls $(\mathrm{N}=$ 24 ), about $24 \%$ of the total. Burseraceae were the second one with 8 kinds of galls, followed by Nyctaginaceae and Sapotaceae, each with 5, and then by Erythroxylaceae, Malpighiaceae and Solanaceae, each with 4 (Tab. II). These results differ from the pattern found by HoUARD (1933) for Central and South Americas, in which there was a remarkable predominance of galls on Leguminosae and Asteraceae. Considering that both these families are well represented in restingas (Leguminosae is the most common plant family in the restingas studied. Compositae is the second one in RC and the fourth one in RBM), this result becomes more relevant.

Most restinga galls were observed on leaves $(\mathrm{N}=63)$, a world pattern already pointed out by MANI (1964). Leaves represent an abundant and constant resource in restinga areas. Lateral and apical buds were the second most attacked plant part $(\mathrm{N}=13)$, followed by inflorescence (including closed flower or flower peduncle) $(\mathrm{N}=9)$ and stem $(\mathrm{N}=9)$, fruit $(\mathrm{N}=4)$ and tendril galls $(\mathrm{N}=1)$ (Tab. III).

In addition to the gall makers, other arthropods were found in the internal chambers of the galls, such as: Pseudoscorpiones, Hymenoptera, Lepidoptera and Diptera. Hymenoptera were the most frequent ones. Collectively they were found 
Table II. List of host plants and number of gall or damage in the restingas of Barra de Maricá (RBM), Itaipuaçu (RI) and Carapebus (RC), Rio de Janeiro State.

\begin{tabular}{|c|c|c|c|c|c|}
\hline \multirow{2}{*}{ Host plants Family } & \multirow{2}{*}{ Species } & \multirow{2}{*}{$\begin{array}{l}\text { Number kinds of gall } \\
\text { or damage }\end{array}$} & \multicolumn{3}{|c|}{ Locality } \\
\hline & & & RBM & RI & $\mathrm{RC}$ \\
\hline Anacardiaceae & Atronium sp. & 1 & 1 & - & - \\
\hline Apocynaceae & Aspidosperma pyricollum & 1 & 1 & - & - \\
\hline Asclepiadaceae & Peplonia asteria & 2 & 1 & 1 & 2 \\
\hline \multirow[t]{2}{*}{ Asteraceae } & Mikania hoehnei & 2 & 2 & 2 & - \\
\hline & Vernonia rufogrisea & 1 & - & - & 1 \\
\hline Bignoniaceae & Arrabidaea conjugata & 3 & 3 & 1 & 2 \\
\hline Boraginaceae & Cordia verbenacea & 3 & 3 & 2 & 2 \\
\hline \multirow[t]{3}{*}{ Burseraceae } & Protium brasiliense & 2 & 2 & - & - \\
\hline & Protium heptaphyllum & 2 & - & - & 2 \\
\hline & Protium icicariba & 4 & - & - & 4 \\
\hline Cactaceae & Selenicereus setaceus & 1 & 1 & 1 & - \\
\hline Celastraceae & Maytenus obtusifolia & 2 & 2 & 1 & 1 \\
\hline Chrysobalanaceae & Couepia ovalifolia & 2 & 2 & - & - \\
\hline \multirow[t]{3}{*}{ Clusiaceae } & Clusia fluminensis & 1 & 1 & - & - \\
\hline & Clusia hilariana & 1 & - & - & 1 \\
\hline & Clusia lanceolata & 1 & 1 & 1 & - \\
\hline Convolvulaceae & Jacquemontia holosericea & 1 & 1 & 1 & - \\
\hline Erythroxylaceae & Erythroxylum ovalifolium & 4 & 4 & 4 & 4 \\
\hline Euphorbiaceae & Sebastiania glandulosa & 3 & - & 3 & 2 \\
\hline Lauraceae & Ocotea notata & 1 & 1 & - & 1 \\
\hline \multirow[t]{3}{*}{ Leguminosae } & Dalbergia ecastophylla & 1 & - & - & 1 \\
\hline & Inga maritima & 1 & 1 & 1 & - \\
\hline & Stylosanthes guianensis & 1 & 1 & - & 1 \\
\hline \multirow[t]{2}{*}{ Loranthaceae } & Psittacanthus dichrous & 1 & 1 & - & - \\
\hline & Struthanthus maricensis & 1 & 1 & - & - \\
\hline \multirow[t]{3}{*}{ Malpighiaceae } & Byrsonima sericea & 3 & 3 & 2 & 3 \\
\hline & Heteropteris nitida & 1 & 1 & - & - \\
\hline & Tetrapteris phlomoides & 1 & 1 & - & - \\
\hline Melastomataceae & Miconia cinnamomifolia & 1 & - & - & 1 \\
\hline Myrsinaceae & Rapanea parvifolia & 1 & 1 & 1 & 1 \\
\hline \multirow[t]{7}{*}{ Myrtaceae } & Eugenia copacabanensis & 2 & 2 & - & - \\
\hline & Eugenia multiflora & 6 & - & - & 6 \\
\hline & Eugenia rotundifolia & 3 & 3 & 3 & - \\
\hline & Eugenia uniflora & 3 & 3 & 1 & 1 \\
\hline & Myrcia ovata & 3 & 3 & - & 3 \\
\hline & Myrciaria floribunda & 3 & 2 & 1 & 3 \\
\hline & Neomitranthes obscura & 4 & 4 & - & 4 \\
\hline Nyctaginaceae & Guapira opposita & 5 & 5 & 4 & 2 \\
\hline Ochnaceae & Ouratea cuspidata & 1 & 1 & - & - \\
\hline Olacaceae & Ximenia americana & 1 & 1 & 1 & - \\
\hline Passifloraceae & Passiflora mucronata & 1 & - & - & 1 \\
\hline Piperaceae & Piper divaricatum & 1 & - & - & 1 \\
\hline \multirow[t]{2}{*}{ Rubiaceae } & Borreria verticillata & 1 & 1 & - & 1 \\
\hline & Diodia gymnocephala & 1 & - & - & 1 \\
\hline Sapindaceae & Paullinia weinmanniaefolia & 3 & 2 & 2 & 3 \\
\hline \multirow[t]{3}{*}{ Sapotaceae } & Manilkara subsericea & 3 & 1 & 1 & 3 \\
\hline & Pouteria caimito & 1 & 1 & 1 & - \\
\hline & Pouteria venosa & 1 & 1 & 1 & - \\
\hline Smilacaceae & Smilax rufescens & 3 & 3 & 1 & 2 \\
\hline \multirow[t]{3}{*}{ Solanaceae } & Aureliana fasciculata & 2 & - & - & 2 \\
\hline & Solanum affine & 1 & 1 & 1 & - \\
\hline & Solanum inaequale & 1 & - & - & 1 \\
\hline Verbenaceae & Lantana camara & 1 & 1 & - & - \\
\hline Total & & 101 & 72 & 39 & 62 \\
\hline
\end{tabular}


in $56 \%$ of the kinds of the galls (Tab. IV). The majority of the Hymenoptera were parasitoids, but some of them were phytophagous. Among the latter, some species were able to modify the gall morphology, such as the inquilinous wasp of conical galls on Paullinia weinmanniaefolia (Sapindaceae). This wasp produces an enlargement of the gall and the development of endogalls (Fig. 86b). These internal protuberances grow and squash the cecidogenous larva, causing its death. Other less remarkable gall modifications due to inquilinous wasps were observed in conical galls on Erythroxylum ovalifolium (Erythorxylaceae) (Fig. 34), cylindrical gall on Eugenia rotundifolia (Fig. 61) and ovoid galls on Myrcia ovata (Myrtaceae) (Fig. $67)$. These modifications cause an enlargement of the gall.

Table III. Distribution of galls in the plant parts at restingas of Barra de Maricá (RBM), Itaipuaçu (RI) and Carapebus (RC), Rio de Janeiro State.

\begin{tabular}{lcc}
\hline \multirow{2}{*}{ Plant part } & \multicolumn{2}{c}{ Kind of gall } \\
\cline { 2 - 3 } & Number & Percentage (\%) \\
\hline Leaf & 63 & 62,4 \\
Bud & 13 & 12,9 \\
Inflorescence (closed flower, flower peduncle) & 9 & 8,9 \\
Stem & 9 & 8,9 \\
Fruit & 4 & 3,9 \\
Tendril & 1 & 1,0 \\
\hline
\end{tabular}

GAGNÉ (1994) indicated that the Pteromalidae, Platygastridae and Torymidae were the most common parasitoids of gall midges. A different result was found in restingas, where Eulophidae and Eurytomidae were the predominant families. FERNANDES et al. (1988) found similar results in their research in Minas Gerais, Brazil. These data suggest that Eulophidae and Eurytomidae may be the most important natural enemies of gall midges in the Neotropical region.

Tritonymphs and adults of Novohorus sp. (pseudoscorpions, probably a new species) were found in cylindrical galls on two different species of Myrtaceae, namely: Eugenia multiflora (in RC) and Eugenia rotundifolia (in RBM). Pseudoscorpions are known by their predaceous habits.

Leptothorax sp. (Formicidae) was found in cylindrical galls on Eugenia rotundifolia. This species invades empty galls and establishes a society there. Trotteria sp., Contarinia sp., Resseliella sp. and Clinodiplosis spp. were found as inquilines in galls yet inhabited by cecidogenous larva. All of them caused the gall maker's death.

Lestodiplosis spp. are known as predators of arthropods. This habit was confirmed in the studied galls, where they preyed upon cecidogenous larvae. Nineteen species of Lasiopteridi and sixty two species of Cecidomyiidi were found. The latter supertribe showed the greatest richness of species, being responsible for $76,5 \%$ of the total of the identified species, with the tribe Asphondyliini the most numerous. The best represented genera of Cecidomyiidae in this research were Clinodiplosis $(\mathrm{N}=10)$, Asphondylia $(\mathrm{N}=7)$, Stephomyia $(\mathrm{N}=6)$, Dasineura $(\mathrm{N}=4)$ and Neolasioptera $(\mathrm{N}=$ 
Table IV. Frequency of Hymenoptera (parasitoids or phytophagous) in galls or plant damage at restingas of Barra de Maricá (RBM), Itaipuaçu (RI) and Carapebus (RC), Rio de Janeiro State.

\begin{tabular}{|c|c|c|}
\hline & Number kinds of gall & Number kinds of gall with Hymenoptera \\
\hline Atronium sp. & 1 & - \\
\hline Aspidosperma pyricollum & 1 & 1 \\
\hline Peplonia asteria & 2 & 2 \\
\hline Mikania hoehnei & 2 & 2 \\
\hline Vernonia rufogrisea & 1 & - \\
\hline Arrabidaea conjugata & 3 & 1 \\
\hline Cordia verbenacea & 3 & 1 \\
\hline Protium braziliense & 2 & 1 \\
\hline Protium heptaphyllum & 2 & 2 \\
\hline Protium icicariba & 4 & 2 \\
\hline Selenicereus setaceus & 1 & - \\
\hline Maytenus obtusifolia & 2 & 1 \\
\hline Couepia ovalifolia & 2 & 1 \\
\hline Clusia fluminensis & 1 & 1 \\
\hline Clusia hilariana & 1 & 1 \\
\hline Clusia lanceolata & 1 & 1 \\
\hline Jacquemontia holosericea & 1 & - \\
\hline Erythroxylum ovalifolium & 4 & 3 \\
\hline Sebastiania glandulosa & 3 & 3 \\
\hline Ocotea notata & 1 & 1 \\
\hline Dalbergia ecastophylla & 1 & 1 \\
\hline Inga maritima & 1 & 1 \\
\hline Stylosanthes guianensis & 1 & 1 \\
\hline Psittacanthus dichrous & 1 & - \\
\hline Struthanthus maricensis & 1 & - \\
\hline Byrsonima sericea & 3 & 2 \\
\hline Heteropteris nitida & 1 & - \\
\hline Tetrapteris phlomoides & 1 & - \\
\hline Miconia cinnamomifolia & 1 & 1 \\
\hline Rapanea parvifolia & 1 & - \\
\hline Eugenia copacabanensis & 2 & 2 \\
\hline Eugenia multiflora & 6 & 5 \\
\hline Eugenia rotundifolia & 3 & 2 \\
\hline Eugenia uniflora & 3 & 3 \\
\hline Myrcia ovata & 3 & 2 \\
\hline Myrciaria floribunda & 3 & 2 \\
\hline Neomitranthes obscura & 4 & 2 \\
\hline Guapira opposita & 5 & 3 \\
\hline Ouratea cuspidata & 1 & - \\
\hline Ximenia americana & 1 & - \\
\hline Passiflora mucronata & 1 & - \\
\hline Piper divaricatum & 1 & - \\
\hline Borreria verticillata & 1 & 1 \\
\hline Diodia gymnocephala & 1 & 1 \\
\hline Paullinia weinmanniaefolia & 3 & 1 \\
\hline Manilkara subsericea & 3 & 3 \\
\hline Pouteria caimito & 1 & 1 \\
\hline Pouteria venosa & 1 & 1 \\
\hline Smilax rufescens & 3 & 2 \\
\hline Aureliana fasciculata & 2 & 1 \\
\hline Solanum affine & 1 & 1 \\
\hline Solanum inaequale & 1 & 1 \\
\hline Lantana camara & 1 & - \\
\hline Total & 101 & $64(=63)$ \\
\hline
\end{tabular}


4). Among the species of Clinodiplosis, 6 were associated with simple galls (marginal rolls), 2 were in complex galls and the others were free-living species, found in fruits and closed flowers. This pattern differs from the one in the Holartic region where the majority of Clinodiplosis species is fungivorous (GAGNE 1989). Concerning to Asphondylia, four species produced flower or fruit galls. The high incidence of this genus in the reproductive parts of the plant had already been noticed by MöHN (1961). All Stephomyia species were associated with Myrtaceae, confirming their specificity to this plant family. Among the species of Dasineura found in the restingas, 2 induced complex galls and the others were found in marginal rolls. Living free in flowers and inducting simple galls are their most common habits in other zoogeographic regions.

The similarity of the gall composition of the restingas was measured by using Sorensen's index. The results showed that RBM is more similar to RI (RBM X RI

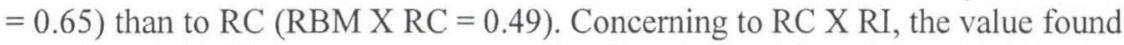
was about 0.47 . These numbers suggest that there is a positive relationship between geographic proximity and fauna similarity.

The geographic distribution of Asphondylia cordiae Möhn, 1959; Camptoneuromyia Felt, 1908; Alycaulus Rübsaamen, 1916 and Proasphondylia guapirae Maia, 1993 were enlarged. Before this study, Asphondylia cordiae had been recorded only in El Salvador (GAGNÉ 1994). The first report of Camptoneuromyia in Brazil is made here. Previous reports were from El Salvador, Colombia, San Vincent and Trinidad. Alycaulus was known to occur in Colombia, Costa Rica, El Salvador and Amazonas (Brazil) (GAGNÉ, 1994). Now, the distribution area of this genus comprises Rio de Janeiro State, too. Proasphondylia guapirae previously recorded only in Restinga of Arraial do Cabo (Rio de Janeiro, Brazil) (MAIA 1993b) has its first report in Maricá.

Eight new genera and fourteen new species of gall midges were found in the restingas (descriptions in preparation). This number of new taxa is a consequence of the scarcity of taxonomic studies on the great diversity of species in neotropical region.

Data on Cecidomyiidae galls or damage in the three studied restingas of Rio de Janeiro State are presented here. They are arranged under host plant species in alphabetical order. Information about the host plants geographic distribution is given, as well as their registration number in the MNRJ herbarium. Regarding to the galls or damage, data include their description, gall maker identification, number of galls per leaf (in case of leaf galls), arthropod fauna associated, number of cecidogenous larvae per gall, examined material and localities and periods and points of gall occurrence.

\section{Data on Cecidomyiidae galls or damage}

\section{Anacardiaceae}

\section{Atronium sp.}

Registration number: 195593 (V. Maia leg.)

Points of occurrence: RBM: MLP - 200, 400, 1400, 3800, 4000, 4200, 4800, 5000,5400 and $5600 \mathrm{~m}$. 
Gall (Fig. 2). Leaf gall, projecting on both surfaces; circular and conspicuous on the superior surface (diameter: $0.3-0.4 \mathrm{~cm}$ ) and permanently open on the inferior surface; glabrous; monothalamous; green or red. Number of galls/leaf: 1-5. Gall maker: Coccoidea. Other arthropods: inquiline-Clinodiplosis sp. (Cecidomyiidae). Pupation in the gall. Number of gall midge larvae/gall: 1. Locality: RBM. Material: Clinodiplosis sp. - 16 larvae (17.X.1987); 1 male (06.VI.1987) and 15 galls (9 on 20.VII.1995, 6 on 12.XII.1997). Periods of gall occurrence: April, June, July, October, December. Points of occurrence: $4800 \mathrm{~m}$. Comments. MONTEIRO et al. (1993) recorded this gall but the plant was misidentified as Protium heptaphyllum.

\section{Apocynaceae}

\section{Aspidosperma pyricollum Muell. Arg.}

Registration number: 194938 (V. Maia leg.)

Distribution: Brazil (Pernambuco, Minas Gerais, Espírito Santo, Rio de Janeiro, São Paulo, Santa Catarina).

Points of occurrence: RBM: MLP - 200, 1000, 1600, 2200, 3600, 3800, 5000-5600m; ZBP - 1800, 3000, 4600, 4800, 5200, 6200m.

Gall (Fig. 3). Leaf gall, projecting on both surfaces; circular on the superior surface (diameter: $0.2-0.3 \mathrm{~cm}$ ) and permanently open on the opposite surface; glabrous; monothalamous; red. Number of galls/leaf: 1-16. Gall maker: Psyliidae. Other arthropods associated: Cecidomyiidi (Cecidomyiidae) (pupation in the gall) and Braconidae (Hymenoptera). Number of gall midge larvae/gall: 1. Locality: RBM. Material: Cecidomyiidi - 1 male (20.X.1986) and 22 galls (19 on 11.VIII. 1990, 3 on 12.XII.1997). Periods of gall occurrence: January, April, May, August, October, December. Points of occurrence: MLP - 200, 1000, 5000, 5200, 5600m; ZBP: 1800, 4600, 4800, 5200m. Comments. RÜBSAAMEN (1907) described a similar Psyliidae gall on Aspidosperma sp.

\section{Asclepiadaceae}

\section{Peplonia asteria (Vell.) Font. \& Schw.}

Registration number: 194939 (V. Maia leg.)

Monotypic genus. Endemic species of restingas. Distribution: Brazil (Bahia, Espírito Santo, Rio de Janeiro).

Points of occurrence: RBM: MLP - 200, 600-1000, 1400-2000, 2600-3000, 3400-3600m; RI: 400-2800, 3200, 3800-4200m; RC: CLP - 200-400, 1400, 1800, 2200, 3000-3400; PGP - 200-1600, 2200-2600m.

Gall (Fig. 4). Leaf roll; length: $0.8-1.0 \mathrm{~cm}$, basal width: $0.2-0.3 \mathrm{~cm}$; glabrous; monothalamous; green or red. Gall maker: Clinodiplosis sp. (Cecidomyiidae). Pupation in the gall. Number of gall midge larvae/gall: 1. Locality: RC. Material: 6 larvae (1 on 28.VIII.1998; 4 on 24.IX.1998 and 1 on 30.I.1999) and 2 galls (29.VI.1998). Periods of gall occurrence: July-August. Points of occurrence: CLP -3200-3400m; PGP - 1000, 3400m.

Gall (Fig. 5). Closed flower; length: $0.6 \mathrm{~cm}$, medial width: $0.4 \mathrm{~cm}$; glabrous; monothalamous; green. Gall maker: Asphondylia sp. (Cecidomyiidae). Pupation in 
the gall. Number of gall midge larvae/gall: 1. Localities: RBM, RI and RC. Material: RBM: 2 females (07.VIII.1998); RC: 7 larvae (6 on 29.VIII.1998 and 1 on 19.XII.1998), 15 pupal exuviae (29.VIII.1998), 7 females (2 on 25.VII.1998, 5 on 29.VIII.1998), 5 males (2 on 25.VII.1998 and 3 on 29.VIII.1998) and 2 galls (25.VII.1998). Other arthropods: parasitoids - Eulophidae and Torymidae (Hymenoptera). Periods of gall occurrence: July-October. Points of occurrence: RBM: MLP - 200, 800, 1400-1600, 2000m; RC: CLP - 200, 2200m; PGP - 200-600, $1000,1400-1600,2200,2600 \mathrm{~m}$.

\section{Asteraceae (= Compositae)}

\section{Mikania hoehnei Robinson}

Registration number: 194975 (V. Maia leg.)

Distribution: Brazil (Rio de Janeiro, São Paulo, Paraná, Santa Catarina).

Points of occurrence: RBM: MLP - 200, 3000, 3200m; ZBP - 600, 1000, $1200,1600,2000,2400,3200-3800,4200,4800,5000,5600 m$. RI: 400, 800, 3600, $4000 \mathrm{~m}$.

Gall (Fig. 6). Leaf roll; length: $1.0 \mathrm{~cm}$, width: $0.3 \mathrm{~cm}$; glabrous; monothalamous; green. Gall maker: Clinodiplosis sp. (Cecidomyiidae). Pupation in the gall. Number of gall midge larvae/gall: 1. Localities: RBM and RI. Material: RBM: 3 larvae (21.VIII.1998), 1 female (07.VIII.1998), 1 gall (05.VI.1998); RI: 1 pupal exuviae (01.VIII.1998), 1 female (01.VIII.1998), 1 gall (12.VI.1998). Other arthropods: Eulophidae (Hymenoptera); Sciaridae (Diptera). Periods of gall occurrence: June-August. Points of occurrence: RBM: ZBP $-600 \mathrm{~m}$. RI: $800 \mathrm{~m}$.

Gall (Fig. 7). Stem swelling; ovoid; length: $1.0-1.8 \mathrm{~cm}$, width: $0.5-0.8 \mathrm{~cm}$; glabrous; monothalamous; coloured like the stem. Gall maker: Alycaulus sp. (Cecidomyiidae). Pupation in the gall. Number of gall midge larvae/gall: 1. Localities: RBM and RI. Material: RBM: 9 females (2 on 10.X.1987, 7 on 07.VIII.1998), 5 galls (III.1998); RI: 3 galls (12.VI.1998). Other arthropods: Dimeromicrus cecidomyiae (Torymidae, Hymenoptera). Periods of gall occurrence: March, April, June, October. Points of occurrence: RBM: MLP - 600, 1000, 2000, 4800, 5600m. RI: 400m. Comments. RÜBSAAMEN (1916) described a similar gall on Mikania sp. from Amazonas (Brazil).

Figs 2-19. Restinga galls. (2) Leaf gall on Atronium sp. (Anacardiaceae); (3) Leaf gall on Aspidosperma pyricollum (Apocynaceae); (4-5) on Peplonia asteria (Asclepiadaceae): (4) rolled young leaf; (5) flower bud gall; (6-7) on Mikania hoehnei (Asteraceae): (6) young leaf roll; (7) stem swelling; (8) bud gall on Vernonia rufogrisea (Asteraceae); (9-10) on Arrabidaea conjugata (Bignoniaceae): (9) conical leaf gall; (10) leaf midvein swelling; (11-13) on Cordia verbenacea (Boraginaceae): (11) globular leaf gall; (12) Inflorescence gall; (13) Petiole swelling; (14-15) on Protium brasiliense (Burseraceae): (14) conical leaf gall on; (15) marginal leaf roll; (16-17) on Protium heptaphyllum (Burseraceae): (16) ovoid leaf gall; (17) marginal leaf roll; (18-19) on Protium icicariba (Burseraceae): (18) conical leaf gall; (19) marginal leaf roll. 
The gall midges from three restingas of Rio de Janeiro...
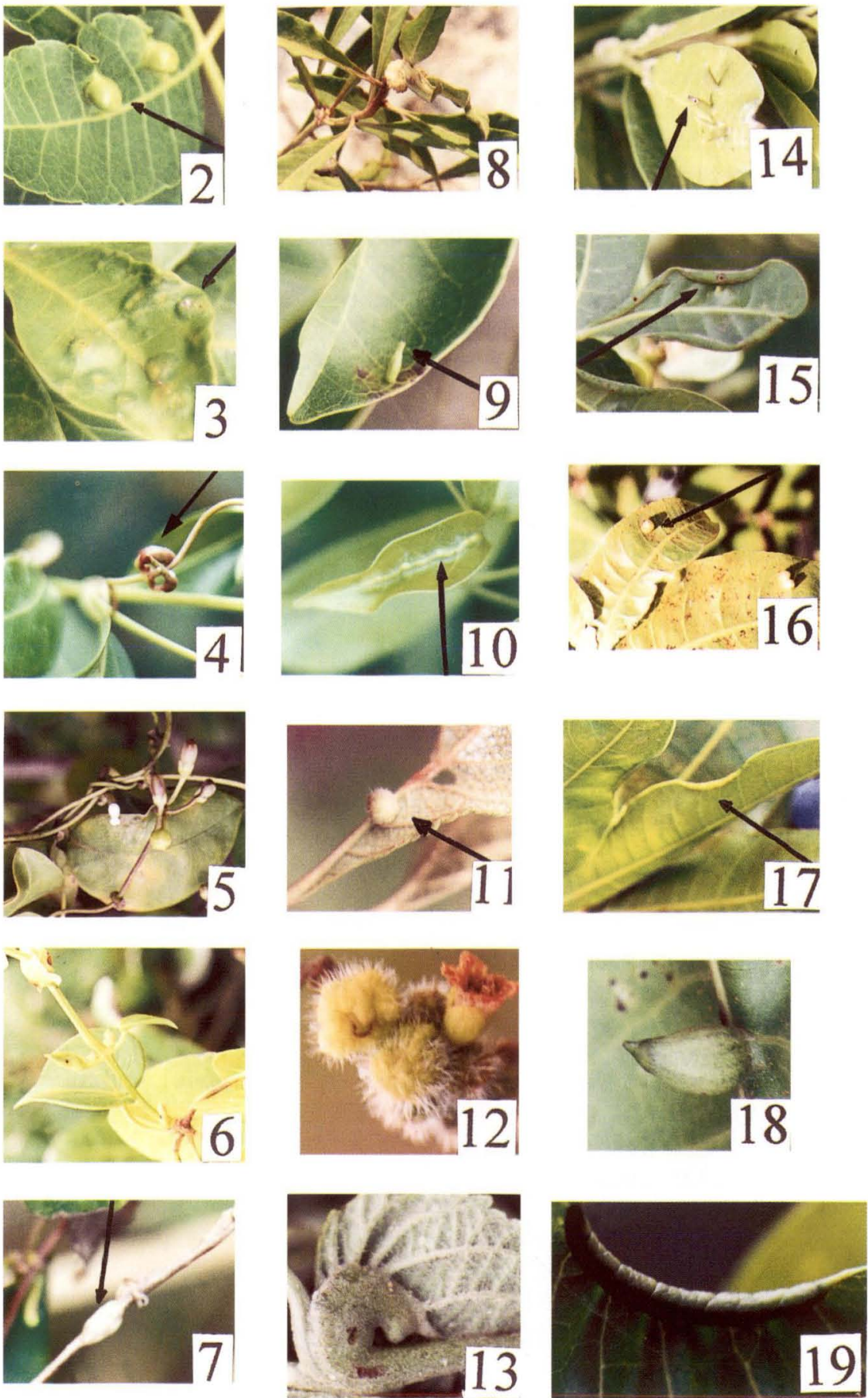

Revta bras. Zool 18 (2): 583 - 629,2001 


\section{Vernonia rufogrisea St. Hill.}

Registration number: 195603 (V. Maia leg.)

Distribution: Brazil (Bahia, Tocantins, Goiás, Minas Gerais, Espírito Santo, Rio de Janeiro, São Paulo).

Points of occurrence: RC: CLP - 200-400, 1400-2000, 2400-2800m; PGP $200,600,1600 \mathrm{~m}$.

Gall (Fig. 8). Spherical bud gall; diameter: $0.3 \mathrm{~cm}$; pubescent; monothalamous; yellowish. Gall maker: Asphondylia sp. (Cecidomyiidae). Pupation in the gall. Number of gall midge larvae/gall: 1. Locality: RC. Material: 1 female (28.IX. 1998), 1 male (28.IX.1998), 7 galls (25.IX.1998). Periods of gall occurrence: September-January. Points of occurrence: same as the host plant.

\section{Bignoniaceae}

\section{Arrabidaea conjugata (Vell.) Mart.}

Registration number: 194951 (V. Maia leg.)

Distribution: from Costa Rica to Brazil (from Amazonas to Paraná).

Points of occurrence: RBM: MLP - 200, 1200, 3000-5000, 5600-6000m; ZBP - 4600-5400, 6000, 64000, 6600m. RI: 200, 400, 1000, 1200, 1800, 3400, 4000m. RC: CLP - 1200, 2000, 2400m.

Gall (Fig. 9). Conical leaf gall; length: $0.8-1.4 \mathrm{~cm}$; basal width: $0.5 \mathrm{~cm}$; glabrous; monothalamous; green. Gall maker: Cecidomyiidi (Cecidomyiidae). Pupation in the gall. Number of gall midge larvae/gall: 1. Localities: RBM, RI and RC. Material: RBM: 5 larvae (2 on 10.X.1998, 3 on 06.XII.1997), 4 pupal exuviae (2 on III.1989, 2 on 25.I.1998), and 21 galls (11 on 12.XII.1997, 10 on II.1998); RI: 2 galls (12.VI.1998); RC: 1 larva (21.III.1999), 1 pupal exuviae (27.II.19999), 1 male (27.II.1999). Other arthropods: parasitoid - Eurytomidae (Hymenoptera). Periods gall of occurrence: January-June, October-December. Points of occurrence: RBM: MLP $-1200,3200,3400,6000 \mathrm{~m}$; ZBP - 5200m. RI: 200m. RC: CLP - 2000m.

Gall (Fig. 10). Stem, tendril or midvein swelling; length: $0.5-0.7 \mathrm{~cm}$; width: 0.2-0.3 cm; glabrous; monothalamous; coloured like the stem, clasper or midvein. Gall maker: Neolasioptera sp. (Cecidomyiidae). Pupation in the gall. Number of gall midge larvae/gall: 1. Localities: RBM and RC. Material: RBM: 7 larvae (1 on 07.VIII.1998, 5 on 27.VII.1998), 6 females (X.1987), 1 male (01.X.1987) and 7 galls (06.XII.1997); RC: 3 larvae (2 on 28.X.1998,1 on 30.I.1999). Periods of gall occurrence: April, October-December. Points of occurrence: RBM: MLP - 4000, 4800m. RC: CLP - 2000m. Comments. TAVARES $(1918,1925)$ described a similar gall on Arrabidaea coleocalix, but he had no success in rearing the gall maker.

Gall. Greatly swollen ovary; spherical; diameter: $1.2 \mathrm{~cm}$; glabrous; monothalamous; purple (as the flower). Gall maker: Cecidomyiinae. Pupation in the gall. Number of gall midge larvae/gall: 1. Locality: RBM. Material: RBM: 2 larvae (1 on XII.1989, 1 on 10.I.1998). Periods of gall occurrence: December, January. Points of occurrence: unknown (material collected by Prof. Maria Célia Rodrigues (Departamento Botânica, MNRJ). 


\section{Boraginaceae}

\section{Cordia verbenacea D. C.}

Registration number: 158517 (Jane G. da Silva leg.)

Distribution: Brazil (from Ceará to Rio Grande do Sul), Paraguay and Argentina.

Points of occurrence: RBM: MLP - 200, 400, 3200, 3600, 3800, 4400-5000, 5800-6400m; ZBP - 800, 1600, 1800, 2200, 2600, 2800, 4600, 4800, 5200-5600, 6200-6600m. RI: 200, 1600, 2000, 2200, 3200, 3400, 3800m. RC: CLP - 200, 3200- 3400m.

Gall (Fig. 11). Globular leaf gall; diameter: $0.5 \mathrm{~cm}$; hairy; monothalamous; green. Number of galls/leaf: 1-17. Gall maker: Cordiamyia globosa Maia, 1996 (Cecidomyiidae). Pupation in the gall. Number of gall midge larvae/gall: 1. Localities: RBM, RI and RC. Material: RBM: 1 male (25.VII.1998), 2 pupal exuviae (25.VII.1998). Other arthropods: parasitoids (Hymenoptera): Pteromalidae - Lyrcus sp. and a new genus and species; Eulophidae - Galeopsomyia sp.; Eurytomidae - Eurytoma sp.; Torymidae - Torymoides sp. and Torymus sp.; Platygastridae Synopeas sp. Periods of gall occurrence: January-June, October-December. Points of occurrence: RBM: MLP - 200, 4400, 4600, 5000m; ZBP - 4600, 5200m, $6200 \mathrm{~m}$. RI: 200, 1600, 2000, 2200, 3200,3400,3800m. RC: the same ones recorded to host plant. Comments. Gall and gall maker described by MAIA (1996a).

Gall (Fig. 12). Closed flower; ovoid; length: $0.5 \mathrm{~cm}$; width: $0.2 \mathrm{~cm}$; hairy; monothalamous; green or yellow. Gall maker: Asphondylia cordiae Möhn, 1975 (Cecidomyiidae). Pupation in the gall. Number of gall midge larvae/gall: 1. Localities: RBM, RI and RC. Material: RBM: 1 larva (05.VI.1998), 4 pupal exuviae (1 on 12.I.1998, 2 on II.1998, 1 on 03.VII.1998), 2 females (II.1998), 6 galls (3 on III.1998, 3 on 05.IV.1998); RI: 2 galls (12.VI.1998). Periods of gall occurrence: January-June. Points of occurrence: RBM: MLP -200, 4600, 5000m; ZBP - 4600, $5200,6200 \mathrm{~m}$. RI: 200, 1600, 2200,3200,3400m. RC: the same ones recorded to host plant. Comments. Gall and gall maker described by MöHN (1975).

Gall (Fig. 13). Leaf petiole or midvein swelling; ovoid; length: $0.3-0.4 \mathrm{~cm}$; basal width: $0.3 \mathrm{~cm}$; apical width: $0.1 \mathrm{~cm}$; glabrous; monothalamous; green. Gall maker: Clinodiplosini (Cecidomyiidae). Pupation in the gall. Number of gall midge larvae/gall: 1. Localities: RBM and RC. Material: RBM: 7 larvae (05.VII.1998); RC: 6 galls (28.VIII.1998). Periods of gall occurrence: June-August. Points of occurrence: RBM: MLP - 200, 400,3600m; RC: the same ones recorded to host plant.

\section{Burseraceae}

\section{Protium brasiliense (Spr.) Engl.}

Registration number: 178083; 195592 (V. Maia leg.)

Distribution: Brazil (common in forests and with some registers in restingas)

Points of occurrence: RBM: ZBP $-600 \mathrm{~m}$.

Gall (Fig. 14). Conical leaf gall; on both leaf surface, but more common on the superior one; length: $0.6-1.0 \mathrm{~cm}$; basal width: $0.1-0.3 \mathrm{~cm}$; glabrous; monotha- 
lamous; green. Number of galls/leaf: 1-13. Gall maker: Cecidomyiidi. Pupation in the gall. Number of gall midge larvae/gall: 1. Locality: RBM. Material: 10 larvae (4 on 25.IV.1998, 6 on 21.VIII.1998), 2 pupal exuviae (23.XII.1998), 3 males (23.XII.1998) and 20 galls (25.IV.1998). Periods of gall occurrence: April-December. Points of occurrence: RBM: ZBP $-600 \mathrm{~m}$.

Gall (Fig. 15). Marginal leaf roll; length: 1.0-1.7 cm; width: $0.1-0.2 \mathrm{~cm}$; glabrous; monothalamous; green. Number of galls/leaf: 1-7. Gall maker: Cecidomyiinae. Pupation in the gall. Number of gall midge larvae/gall: 1. Locality: RBM. Material: 1 larva (25.IV.1980 and 5 galls (25.IV.1998). Other arthropods: parasitoid - Hymenoptera. Periods of gall occurrence: April-June. Points of occurrence: RBM: ZBP - 600m.

\section{Protium heptaphyllum (Aublet.) March}

Registration number: 194959 (V. Maia leg.)

Distribution: South America (Colombia, Venezuela, Guyana, Suriname, French Guyana, Brazil, Bolivia, Paraguay) and Trinidad. In Brazil, it occurs in nearly all states, except in Santa Catarina and Rio Grande do Sul.

Points of occurrence: RC: CLP - 200-400, 1200-2800, 4200m; PGP -2400, $3200 \mathrm{~m}$.

Gall (Fig. 16). Ovoid leaf gall; length: $0.3-0.4 \mathrm{~cm}$; medial width: $0.2-0.3 \mathrm{~cm}$; glabrous; monothalamous; green. Number of galls/leaf: 4-15. Gall maker: Cecidomyiidi. Pupation in the gall. Number of gall midge larvae/gall: 1. Locality: RC. Material: 2 pupal exuviae (1 on 27.XI. 1998, 1 on 17.III.1999), 1 female (17.III. 1999), 2 males (1 on 29.VI.1998, 1 on 27.XI.1998). Other arthropods: parasitoid Hymenoptera. Periods of gall occurrence: May-March. Points of gall occurrence: CLP - 200-400, 1200-1400, 1800-2000, 2400-2800m; PGP-2400m.

Gall (Fig. 17). Marginal leaf roll; length: 1.0-1.7 cm; width: 0.1-0.2 cm; glabrous; monothalamous; green. Number of galls/leaf: 1-7. Gall maker: Cecidomyiidi. Pupation in the gall. Number of gall midge larvae/gall: 1. Locality: RC. Material: 4 larvae ( 1 on 29.VI.1998, 3 on 21.VI.1998), 5 pupal exuviae (1 on 29.VI.1998, 1 on 29. VII.1998, 2 on 28.IX.1998, 1 on 27.II.1999), 11 galls (23.V.1998). Other arthropods: parasitoid - Hymenoptera. Periods of gall occurrence: June-February. Points of occurrence: CLP - 200-400, 1200-2800, 4200m. Comments. Gall described by TAVARES (1922).

\section{Protium icicariba (DC.) March.}

Registration number: 184289 (A. Souza leg.)

Distribution: Brazil (from south Bahia to Rio de Janeiro).

Points of occurrence: RC: CLP - 400-800, 1400-1600, 2000, 2400m, 30003200, 3600-4200m; PGP - 400-600, 1000-3400m.

Gall (Fig. 18). Conical leaf gall; length: $0.6-1.0 \mathrm{~cm}$; basal width: $0.1-0.3 \mathrm{~cm}$; glabrous; monothalamous; green. Number of galls/leaf: 1-13. Gall maker: Cecidomyiinae. Pupation in the gall. Number of gall midge larvae/gall: 1. Locality: RC. Material: 12 larvae (3 on 29.VI.1998, 1 on 24.VII.1998, 1 on 29.VIII.1998, 2 on 24.X.1998, 3 on 27.XI.1998, 1 on 26.II.1999, 1 on 17.III.1999), 3 pupal exuviae (1 
on 28.XI. 1998, 2 on 19.XII.1998), 2 pupae (24.X.1998). Other arthropods: parasitoids - Hymenoptera. Periods of gall occurrence: May-February. Points of gall occurrence: CLP - 400-600, 1400-1600, 2000, 2400, 3000-3200, 3600-4200m; PGP $-400-600,1000-3200 \mathrm{~m}$.

Gall (Fig. 19). Marginal leaf roll; length: 1.0-1.7 cm; width: 0.1-0.2 cm; glabrous; monothalamous; green. Number of galls/leaf: 1-7. Gall maker: Cecidomyiidi. Pupation in the gall. Number of gall midge larvae/gall: 1. Locality: RC. Material: 1 pupal exuviae (27.XI.1998). Other arthropods: parasitoid - Hymenoptera. Periods of gall occurrence: April-February. Points of occurrence: PC - 400600, 1400-1600, 2000, 2400m; PG - 400-600, 1000, 1600-1800, 2200-3200m.

Gall (Fig. 20). Fruit gall; kidney shaped (lateral view), enlarged basally and tapered apically; length: $0.5-0.7 \mathrm{~cm}$; basal width: $0.6 \mathrm{~cm}$; glabrous; monothalamous; green or red. Gall maker: Cecidomyiinae. Pupation in the gall. Number of gall midge larvae/gall: 1. Locality: RC. Material: 4 larvae (25.IX.1998), 2 pupal exuviae (29.XII.1998), 8 galls (24.V.1998). Periods of gall occurrence: May-July, September, December. Points of occurrence: CLP $-400 \mathrm{~m}$; PGP $-600,1000,3000 \mathrm{~m}$.

Gall. Ovoid leaf gall; length: $0.3-0.4 \mathrm{~cm}$; medial width: $0,2-0.3 \mathrm{~cm}$; glabrous; monothalamous; green. Number of galls/leaf: 4-15. Gall maker: Cecidomyiinae. Pupation in the gall. Number of gall midge larvae/gall: 1. Locality: RC. Periods of gall occurrence: May-October. Points of occurrence: CLP - 400, 2800m; PGP - 2400m.

\section{Cactaceae}

\section{Selenicereus setaceus (SD) Berg (= Cereus setaceus)}

Registration number: 146487 (J. Cardoso leg.)

Distribution: Bolivia, Paraguay, Argentina, Brazil (Minas Gerais, Mato Grosso, Rio de Janeiro).

Points of occurrence: RBM: all points of collecting; RI: 200-4500m; RC: CLP - 200-400, 1800, 2400m.

Gall (Fig. 21). Stem swelling; ovoid; length: $2.5-3.0 \mathrm{~cm}$; width: $2.5 \mathrm{~cm}$; glabrous; polithalamous; green. Gall maker: Neolasioptera cerei Rübsaamen, 1905 (Cecidomyiidae). Pupation in the gall. Number of gall midge larvae/chamber: 1 . Localities: RBM and RI. Material: RI: 3 galls (24.IV.1998). Periods of gall occurrence: April. Points of gall occurrence: RBM: MLP $-1600 \mathrm{~m}$; RI: $1000 \mathrm{~m}$. Comments. Gall and gall maker described by RüBSAAMEN (1905).

\section{Celastraceae}

\section{Maytenus obtusifolia Mart. var. obovata Mart.}

Registration number: 194964, 194965 (V. Maia leg.)

Distribution: Brazil (Pará, Ceará, Rio Grande do Norte, Sergipe, Bahia, Espírito Santo, Rio de Janeiro, São Paulo).

Points of occurrence: RBM: MLP-400, 800, 1000, 1400-2200, 2600-4000, 4400-5800m; ZBP: 200-6200m. RI: 200-1000, 1800, 2200, 2800-3600, 4000m. RC: CLP- 200-400, 2200-2400, 4200m; PGP - 200, 800, 1600, 2200-2600, 3000m. 
Gall (Fig. 22). Circular leaf gall, projected on both surfaces; diameter: 0.6-0.7 cm; glabrous; monothalamous; green or yellow. It is a remarkable gall due to its hard walls. Number of galls/leaf: 1-25. Gall maker: Oligotrophini (Cecidomyiidae). Pupation in the gall. Number of gall midge larvae/gall: 1 . Localities: RBM, RI and RC. Material: RBM: 3 males (2 on 20.XII.1986, 1 on 31.III. 1987), 3 females (1 on 20.XII.1986; 1 on 01.XI.1991, 1 on 08.VI.1992), 8 pupal exuviae (2 on 01.XI.1991, 6 on 20.VII.1995), 50 galls (6 on 11.VIII.1990, 44 on 22.III.1997); RI: 28 galls (03.IV.1998); RC: 1 gall (24.V.1998). Other arthropods: parasitoid - Tetrastichinae, n. gen. (Hymenoptera). Periods of gall occurrence: January, March-August, October-December. Points of gall occurrence: the same ones recorded by host plant.

Gall (Fig. 23). Fruit gall; ovoid; length: 0.8-0.9 cm; width: $0.7 \mathrm{~cm}$; glabrous; polithalamous; red. Gall maker: Bruggmanniella maytenuse (Maia \& Couri, 1992). Pupation in the gall. Number of gall midge larvae/chamber: 1. Locality: RBM. Material: 1 male (20.XII.1986), 1 female (13.IX.1986), 2 pupal exuviae (1 on 20.XII.1986, 1 on 17.XII.1987), 6 larvae (14.VIII.1997), 8 galls (07.VIII.1998). Periods of gall occurrence: August-September, December. Points of occurrence: MLP: $2600 \mathrm{~m}$. Comments. Gall and gall maker described by MAIA et al. (1992).

\section{Chrysobalanaceae}

\section{Couepia ovalifolia (Schott) Benth.}

Registration number: 194988 (V. Maia leg.)

Distribution: Brazil (from Pernambuco to Rio de Janeiro).

Points of occurrence: RBM: MLP - 400-800, 1400-1600m.

Gall (Fig. 24). Circular leaf gall, projected on both surfaces; diameter: 0.4 cm; glabrous; monothalamous; green. Number of galls/leaf: 1-16. Gall maker: Dasineura sp. (Cecidomyiidae). Pupation in the gall. Number of gall midge larvae/gall: 1. Locality: RBM. Material: RBM: 3 pupal exuviae (05.VI.1998), 1 pupa (05.VI.1998), 2 males (1 on 15.XII.1989, 1 on 05.VI.1998), 7 females (2 on 02.XII.1989, 1 on 15.XII.1989, 4 on 05.VI.1998), 24 galls (22.III.1998). Periods of gall occurrence: March-June, December. Points of gall occurrence: the same ones recorded by host plant.

Gall (Fig. 25). Marginal leaf roll; length: 0.8-1.5 cm; width: 0.1-0.3 cm; glabrous; monothalamous; green. Number of galls/leaf: 1-10. Gall maker: Lopesiini (Cecidomyiidae). Pupation in the gall. Number of gall midge larvae/gall: 1. Locality:

Figs 20-34. Restinga galls or damage. (20) on Protium icicariba (Burseraceae): fruit gall; (21) Stem swelling on Selenicereus setaceus (Cactaceae); (22-23) on Maytenus obtusifolia (Celastraceae): (22) leaf gall; (23) fruit gall; (24-25) on Couepia ovalifolia (Chrysobalanaceae): (24) circular leaf; (25) marginal leaf roll; (26-28) galls on Clusiaceae: (26) leaf gall on Clusia fluminensis; (27) leaf gall on Clusia hilariana; (28) leaf gall on Clusia lanceolata; (29) flower bud on Jacquemontia holosericea (Convolvulaceae); (30-34) on Erythroxylum ovalifolium (Erythroxylaceae): (30) triangular leaf gall; (31) flower bud gall; (32) rolled young leaf; (33) conical bud gall; (34) modified bud gall. 


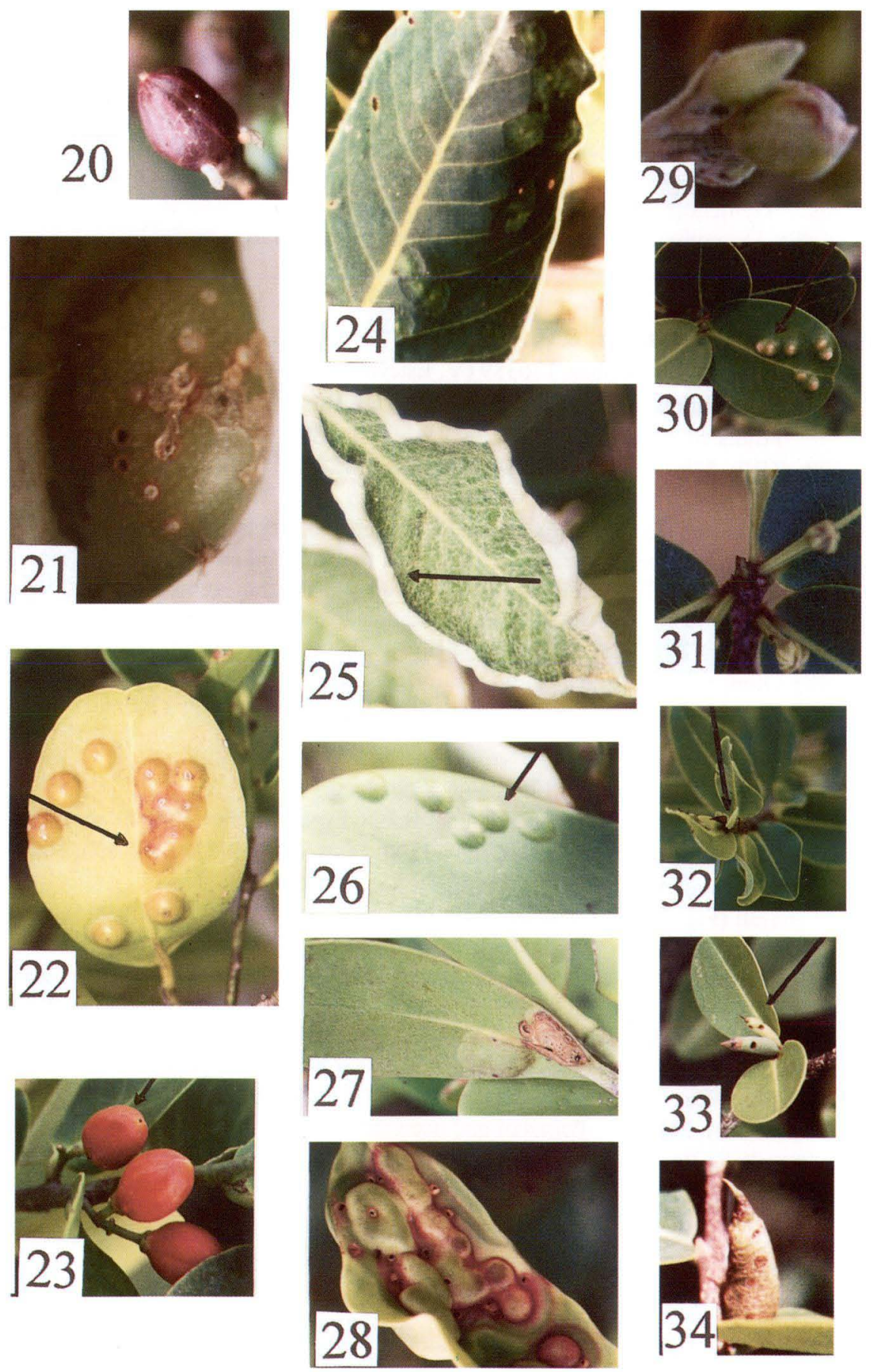


RBM. Material: 34 larvae (7 on 12.III.1988, 15 on 02.XII.1989, 1 on II.1998, 9 on III.1998), 12 pupal exuviae (1 on II.1988, 1 on 01.III.1988, 3 on 02.XII.1989, 2 on 14.VIII.1997, 6 on 17.VIII.1997), 2 pupae (1 on XII.1989, 1 on II.1998), 18 females ( 1 on III.1988, 11 on 02.XII.1989, 6 on 12.XII.1989), 14 males ( 1 on III.1998, 1 on VII.1988, 4 on 17.VII.1988, 1 on 12.IX.1988, 1 on X.1989, 5 on 02.XII.1989, 1 on II.1998), 33 galls (5 on 22.III.1998, 28 on 05.IV.1998). Other arthropods: parasitoids - Hymenoptera. Periods of gall occurrence: February-April, July-August, October, December. Points of gall occurrence: the same ones recorded by host plant.

\section{Clusiaceae}

\section{Clusia fluminensis Tr. \& PI.}

Registration number: 176389 (V. Esteves leg.)

Distribution: Brazil (Bahia, Minas Gerais, Rio de Janeiro).

Points of occurrence: RBM: MLP - 400, 1800, 3800, 4400, 5000, 54005600; ZBP: 2800, 3200-5000, 5400-5800m. RI: 400, 1000, 3200-3400, 4000, $4500 \mathrm{~m}$.

Gall (Fig. 26). Circular leaf gall; diameter: $0.5 \mathrm{~cm}$; glabrous; monothalamous; green. Number of galls/leaf: 1-12. Gall maker: Asphondyliini, near Zalepidota Rübsaamen, 1907 (Cecidomyiidae). Pupation in the gall. Number of gall midge larvae/gall: 1. Locality: RBM. Material: 8 pupal exuviae (2 on 14.VIII.1997, 6 on 17.VIII.1997), 7 females (14.VIII.1997), 7 galls (3 on 30.IV.1990, 4 on 05.IV. 1998). Other arthropods: parasitoid - Eurytomidae (Hymenoptera). Periods of gall occurrence: March-June, August. Points of gall occurrence: ZBP: $3200 \mathrm{~m}$.

\section{Clusia hilariana Schltdl.}

Registration number: 179204 (A. Souza leg.)

Distribution: Brazil (Pernambuco, Bahia, Espírito Santo, Rio de Janeiro).

Points of occurrence: RC: CLP - 1200, 1600-3800m; PGP: 200-600, 1000$3000,3400 \mathrm{~m}$.

Gall (Fig. 27). Circular leaf gall; diameter: $1.0-1.5 \mathrm{~cm}$; glabrous; monothalamous; green. Number of galls/leaf: 1-7. Gall maker: Cecidomyiidi (Cecidomyiidae). Pupation in the gall. Number of gall midge larvae/gall: 1. Locality: RC. Material: 4 larvae (1 on 25.VII.1998, 2 on 27.XI.1998, 1 on 26.II.1999), 8 pupal exuviae (6 on 31.X.1998, 2 on 25.VIII.1998), 5 males (1 on 25.VIII.1998, 1 on 25.IX.1998, 3 on 31.X.1998), 3 galls (24.V.1998). Other arthropods: parasitoids Eupelmidae, Eurytomidae and Platygastridae (Hymenoptera). Periods of gall occurrence: May-February. Points of gall occurrence: CLP-1200, 1600-2000, 2400, 2800m; PGP: 200-600, 1000, 1400-3000, 3400m.

\section{Clusia lanceolata Camb.}

Registration number: 129511 (M. C. Viana leg.)

Distribution: Brazil (Rio de Janeiro, São Paulo).

Points of occurrence: RBM: MLP - 400, 800-1200, 1600, 2000-3000, 3400-3800, 4400-4600, 5000, 5400-5600m; ZBP-200-600, 1400-6600m; RI: 400, $800,3400-4000 \mathrm{~m}$. 
Gall (Fig. 28). Leaf blister; diameter: 0.6-1.1 cm; glabrous; monothalamous; green or red. Number of galls/leaf: 1-18. Gall maker: Chisiamyia nitida Maia, 1996 (Cecidomyiidae). Pupation in the gall. Number of gall midge larvae/gall: 1. Localities: RBM and RI. Material: RBM: 8 larvae (4 on 20.VII.1995, 2 on 28.II.1996, 1 on II.1998, 1 on III.1998), 1 female (11.VIII.1990), 6 galls (3 on 11.VIII.1990, 3 on 05.IV.1998); RI: 5 galls (03.IV.1998). Other arthropods: parasitoid - Inostemma sp. (Platygastridae, Hymenoptera). Periods of gall occurrence: all months of the year. Points of gall occurrence: the same ones recorded to host plant. Comments. Gall and gall maker described by MAIA (1996b).

\section{Convolvulaceae}

\section{Jacquemontia holosericea (Weinman) O'Donell}

Registration number: 168773 (M. Alves leg.)

Distribution: Brazil (Ceará, Rio de Janeiro, São Paulo, Santa Catarina).

Points of occurrence: RBM: MLP - 200-400, 1000, 3800m; ZBP - 600m; RI: 400, 800, 3600, 4000m.

Closed flower (Fig. 29) (length: $1.0 \mathrm{~cm}$; width: $0.4 \mathrm{~cm}$ ) with 1, 2 or 3 gall midge larvae. Cecidomyiidae associated: Schizomyia sp., Camptoneuromyia sp. Pupation in the closed flower. Localities: RBM and RI. Material: RI: Schizomyia sp. - 2 larvae (04.IX.1998), 1 pupal exuviae (01.VIII.1998), 4 females (3 on 02.X.1998, 1 on 01.VIII.1998), 5 males (2 on 01.VIII.1998, 3 on 02.X.1998); Camptoneuromyia sp. -5 females (02.X.1998); 6 attacked closed flower (10.VII. 1998). Periods of occurrence: July-August, December. Points of occurrence: RBM: ZBP $-600 \mathrm{~m}$. RI: the same ones recorded to host plant.

\section{Erythroxylaceae}

\section{Erythroxylum ovalifolium Peyr.}

Registration number: 195003, 195004 (V. Maia leg.)

Distribution: Brazil (Minas Gerais, Rio de Janeiro).

Points of occurrence: RBM: MLP - 400-4600m; ZBP - 200-600, 1400, 1800-6600m; RI: 200-800, 3200-3800m; RC: CLP - 200-600, 1200-1400, $1800-$ 2400, 2800-4200m; PGP - 400, 1000-2400, 2800-3000, 3400m.

Gall (Fig. 30). Triangular leaf gall; length: $0.2 \mathrm{~cm}$; basal width: $0.1 \mathrm{~cm}$; glabrous, monothalamous, greenish. Number of galls/leaf: 1-23. Gall maker: Lasiopteridi (Cecidomyiidae). Pupation in the soil. Number of gall midge larvae/gall: 1. Localities: RBM, RI and RC. Material: RBM: 6 females (14.III.1998), 35 galls (8 on 11.VIII.1990, 27 on 05.IV.1998); RI: 2 females ( 1 on 12.IV.1998, 1 on 03.VII.1998). Other arthropods: parasitoids - Tetrastichinae, n. gen. (Eulophidae) and Platygastridae (Hymenoptera). Periods of gall occurrence: January-December. Points of occurrence: RBM: MLP -2000, 3200-4400, 6200m; ZBP - 200-800, 1400-2000, 2400-3000, 3400-6200m. RI: 400-600, 3400m. RC: CLP - 1200, 1800, 2800m; PGP - 1400, 2200-2400, 2800-3000m. 
Gall (Fig. 31). Closed flower; ovoid; length: $0.2 \mathrm{~cm}$; width: $0.1 \mathrm{~cm}$; glabrous, monothalamous, greenish. Gall maker: Asphondylia sp. (Cecidomyiidae). Pupation in the gall. Number of gall midge larvae/gall: 1. Localities: RBM, RI and RC. Material: RBM: 2 larvae (08.IX.1998), 30 pupal exuviae (23 on 11.VIII.1997, 3 on 01.IX.1997, 1 on 28.X.1997, 1 on 12.I.1998, 2 on II.1998), 5 females (3 on VIII.1997, 2 on II.1998), 8 males (5 on 11.VIII.1997, 2 on 01.IX.1997, 1 on 28.IX.1997), 32 galls (16 on 11.VIII.1990, 16 on 17.I.1998); RI: 1 pupa (01.VIII. 1998); RC: 12 pupal exuviae (10 on 29.VIII.1998, 2 on 26.IX.1998). Periods of gall occurrence: May, August-October, January-February. Other arthropods: parasitoid - Eurytomidae (Hymenoptera). Points of occurrence: RBM: MLP - 400, 5400m. RC: CLP - 200-600, 1600, 3200m; PGP - 600, 1000, 2200m. Comments. MöHN (1959) described galls in fruits induced by Asphondylia erythroxylis on Erythroxylum mexicanum.

Gall (Fig. 32). Rolled young leaf; length: $0.8-1.0 \mathrm{~cm}$; width: $0.2-0.3 \mathrm{~cm}$; glaborus, monothalamous; green. Cecidomyiidae associated: Clinodiplosis sp. and Stomatosematidi cfr. Pupation in the gall. Number of gall midge larvae/gall: 1 . Localities: RBM, RI and RC. Material: RBM: Clinodiplosis sp.: RBM: 4 larvae (21.XI.1997); Stomatosematidi cfr.: RC: 2 males (19.XII.1998). Periods of gall occurrence: November-January. Points of occurrence: RBM: MLP $-400 \mathrm{~m}$, RI: 600m. RC: CLP - 200-600m.

Gall (Fig. 33). Conical bud gall; length: 0.9-1.1 cm; width: 0,1-0,3 cm; glabrous; monothalamous and almost entirely green, except the apex which is brown. Gall maker: Cecidomyiinae. Pupation in the gall. Number of gall midge larvae/gall: 1. Localities: RBM, RI and RC. Material: RBM: 7 larvae ( 3 on 10.X.1997, 4 on 21.IX.1997); 2 pupal exuviae (1 on 20.IX.1987, 1 on 21.XI.1997); 3 pupae (1 on 21.IX.1987, 2 on 01.IX.1997); 3 females ( 1 on 22.IX.1987, 1 on 29.IX.1987, 1 on 21.IX.1997); 11 galls (1 on 10.X.1997, 1 on 12.XII.1997, 7 on 24.IV.1998, 2 on 25.IV.1998); RI: 4 galls (12.VI.1998). Other arthropods: a phytophagous species of Tetrastichinae (Eulophidae: Hymenoptera). Other material: galls modified by Tetrastichinae sp. (Fig. 34) - RBM: 3 galls (17.IV.1998); RI: 2 galls (12.VI.1998). Periods of gall occurrence: March-July, September-December. Points of occurrence: RBM: MLP -400, 2000, 3200, 4200, 4600-4800m; ZBP: 600, 2600, 3400, 4800, 5200-5400, 5800m. RC: CLP-2000, 3000m.

\section{Euphorbiaceae}

\section{Sebastiania glandulosa (Mart.) Pax.}

Registration number: 145928 (J. G. da Silva leg.)

Distribution: Brazil (Minas Gerais, Rio de Janeiro, Mato Grosso, Mato Grosso do Sul, Goiás).

Points of occurrence: RBM: MLP - 1800-2400m; RI: 1800-2200, 2800m; RC: CLP - 1400-1600, 2200-2400, 3400m.

Gall (Fig. 35). Conical bud gall; length: 0.5-0.8 cm; width: 0.1-0.2cm; glabrous, monothalamous, red. Gall maker: Cecidomyiinae. Pupation in the gall. Number of gall midge larvae/gall: 1. Localities: RI and RC. Material: RC: 1 larva 
(26.IX.1998), 3 pupal exuviae (2 on 29.VI.1998, 1 on 28.XI.1998), 1 female (24.VII.1998), 2 galls (29.VI.1998). Other arthropods: Hymenoptera (parasitoid). Periods of gall occurrence: June-July, September-February. Points of occurrence: RI: $2800 \mathrm{~m}$. RC: PGP $-2200-2400 \mathrm{~m}$.

Gall (Fig. 36). Spherical bud gall; diameter: $0.5 \mathrm{~cm}$; glabrous, monothalamous, green or yellow. Gall maker: Schizomyia sp. (Cecidomyiidae). Pupation in the soil. Number of gall midge larvae/gall: 1. Localities: RBM and RC. Material: RBM: 2 males (col.: 08.IX.1998; emerg.: 28.I.1999); RI: 6 galls (10.VII.1998). Other arthropods: Hymenoptera (parasitoid). Periods of gall occurrence: July, September-January. Points of occurrence: RBM: MLP - 1600-2400m; RI: 18002200m. RC: PGP - 2200-2400m.

Gall (Fig. 37). Marginal leaf roll; length: $0.6-0.7 \mathrm{~cm}$; medial width: $0.1 \mathrm{~cm}$; glabrous, monothalamous, green. Gall maker: Cecidomyiidi. Pupation in gall. Number of gall midge larvae/gall: 1. Localities: RI and RC. Material: RI: 3 males (2 on 10.VII.1998, 1 on 02.X.1998); RC: 9 males (28.XI.1998), 6 galls (26.IX. 1998). Other arthropods: Hymenoptera (parasitoid). Periods of gall occurrence: July, SeptemberNovember. Points of occurrence: RI: $1800,2200 \mathrm{~m}$. RC: PGP $-2200 \mathrm{~m}$.

\section{Lauraceae}

\section{Ocotea notata (Ness) Mez.}

Registration number: 195058 (V. Maia leg.)

Distribution: Brazil (Pernambuco, Alagoas, Espírito Santo, Rio de Janeiro).

Points of occurrence: RBM: MLP- all points of collecting; ZBP - 200-2000, 2400, 3400, 4200-4400, 5000, 5600-6000m. RI: 3200, 3800m. RC: CLP-200-400, 1400-2400, 2800-4200m; PGP: 200-800, 1000, 1400-3000, 3400m.

Gall (Fig. 38). Bud gall, ovoid and with an apical spine-like projection; length: $0.7 \mathrm{~cm}$ (including the apical projection); width: $0.4 \mathrm{~cm}$; glabrous, monothalamous; green. Gall maker: Cecidomyiidi. Pupation in the gall. Number of gall midge larvae/gall: 1. Localities: RBM and RC. Material: RBM: 1 larva (07.VIII. 1998), 3 galls (23.V.1998); RC: 4 larvae (1 on 24.V.1998, 2 on 27.II.1999, 1 on 17.III.1999). Other arthropods: Hymenoptera (parasitoid). Periods of gall occurrence: May-July, January-February. Points of occurrence: RBM: MLP - 600m. RC: CLP -200-400, 14002400, 2800-3000, 3800m; PGP - 200-600, 1000, 2400, 3000m.

\section{Leguminosae (= Fabaceae)}

\section{Dalbergia ecastophylla L. Taub.}

Registration number: 195076 (V. Maia leg.)

Distribution: wide spread throughout the tropics of the Old and New World. In the Neotropical region: from Florida (USA) to the South of Brazil, reaching Santa Catarina.

Points of occurrence: RC: CLP - 200-400m.

Gall (Fig. 39). Discoid leaf gall; diameter: $0.4 \mathrm{~cm}$; glabrous, monothalamous; green. It occurs only on the leaf inferior surface. Number of galls/leaf: 3-12. Gall 
maker: Cecidomyiidi (Cecidomyiidae). Pupation in the gall. Number of gall midge larvae/gall: 1. Locality: RC. Material: 6 larvae (3 on 28.VI.1998, I on 29.VI.1998, 1 on 27.XI.1998), 8 pupal exuviae (7 on 28.VI.1998, 1 on 27.XI.1998); 8 females (2 on 28.VI.1998, 2 on 29.VI.1998, 4 on 27.XI.1998), 11 males (3 on 28.VI.1998, 4 on 29.IV.1998, 1 on 25.IX.1998, 1 on 31.X.1998, 2 on 27.XI.1998), 12 galls (23.V.1998). Periods of gall occurrence: April-July, October-November. Points of occurrence: CLP - 200-400m. Comments. Gall described by TAVARES (1922) and illustration showed by HOUARD (1933).

\section{Inga maritima Benth.}

Registration number: 195075 (V. Maia leg.)

Distribution: Brazil (Southeast region).

Points of occurrence: RBM: ZBP - 600m; RC: CLP - 1400-1600m.

Gall (Fig. 40). Stem, petiole or midvein swelling; length: $0.7 \mathrm{~cm}$; width: 0.1 $\mathrm{cm}$; glabrous, monothalamous; green. Number of galls/leaf: 1-4. Gall maker: Neolasioptera sp. (Cecidomyiidae). Pupation in the gall. Number of gall midge larvae/gall: 1. Localities: RBM and RI. Material: RBM: 5 larvae (3 on 15.V.1998, 2 on 21.VIII.1998), 6 pupal exuviae (2 on 15.V.1998, 1 on 05.VI.1998, 1 on 03.VII.1998, 2 on 21.VIII.1998); 4 females (2 on 15.V.1998, 1 on 03.VII.1998, 1 on 21.VIII.1998), 4 males (21. VIII.1998), 7 galls (25.IV.1998); RI: 1 larva (04.IX.1998), 1 pupal exuviae (04.IX.1998), 5 galls (12.VI.1998). Other arthropods: parasitoid - Eupelmidae (Hymenoptera). Periods of gall occurrence: April-August, September. Points of occurrence: RBM: ZBP $-600 \mathrm{~m}$; RI: $400 \mathrm{~m}$. Comments. Similar galls of Neolasioptera ingae were described by TAVARES (1920) on Inga sp. Also MöHN (1964) described others on Inga leptoloba and I. spuria.

\section{Stylosanthes guianensis Sw.}

Registration number: 195077 (V. Maia leg.)

Distribution: wide spread in Tropical America.

Points of occurrence: RBM: MLP-3000m; RC: CLP-200-400, 1200-2000, 2400-2800, 4200m; PGP - 200-600, 1000, 1400-1600, 2200, 2600, 3000m.

Larvae in the inflorescence (Fig. 41): Lestodiplosis sp. Pupation in the inflorescence. Localities: RBM and RC. Material: RBM: 3 females (23. XII.1998), 2 males (23.XII.1998); RC: 7 larvae (6 on 28.VIII.1998, 1 on 25.IX.1998), 6 pupal exuviae (29.VIII.1998), 6 females (5 on 25.IX.1998, 1 on 28.VIII.1998), 6 males

Figs 35-56. Restinga galls or damage. (35-37) on Sebastiania glandulosa (Euphorbiaceae): (35) conical bud gall; (36) spherical bud gall; (37) marginal leaf roll; (38) bud gall on Ocotea notata (Lauraceae); (39-41) on Leguminosae: (39) leaf gall on Dalbergia ecastophylla; (40) petiole swelling on Inga maritima; (41) inflorescence of Stylosanthes guianensis; (42) leaf gall on Psittacanthus dichrous (Loranthaceae); (43-48) on Malpighiaceae: (43-45) on Byrsonima sericea: (43) inflorescence gall; (44) leaf gall; (45) stem swelling; (46) flower bud of Heteropteris nitida; (47) rosette bud gall on tetrapteris phlomoides; (48) bud gall on Miconia cinnamomifolia (Melastomataceae); (49) leaf gall on Rapanea parvifolia (Myrsinaceae); (50-56) on Myrtaceae - (50-51) on Eugenia copacabanensis: (50) spiral leaf gall; (51) fusiform leaf gall; (52-65) on Eugenia multiflora: (52) cylindrical stem gall; (53) circular leaf gall; (54) marginal leaf roll; (55) pyriform leaf gall; (56) claviform leaf gal. 

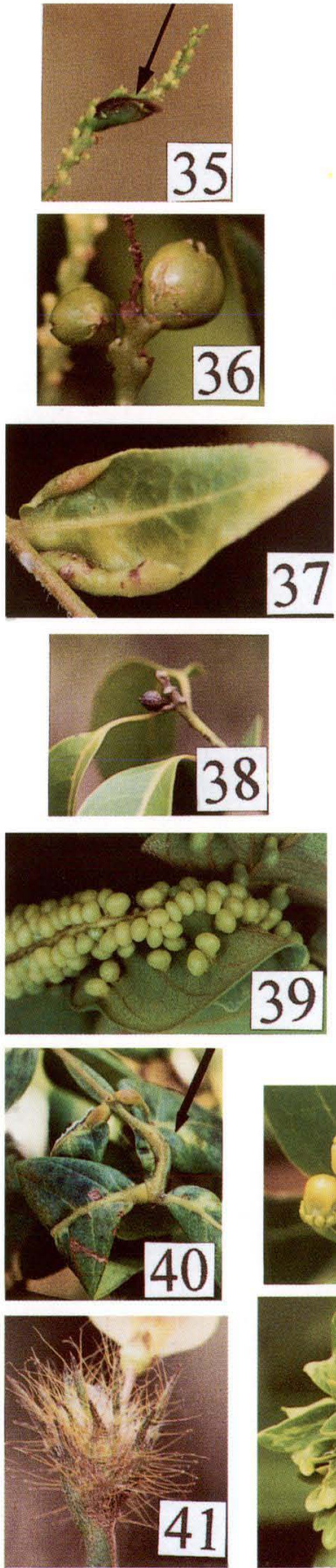
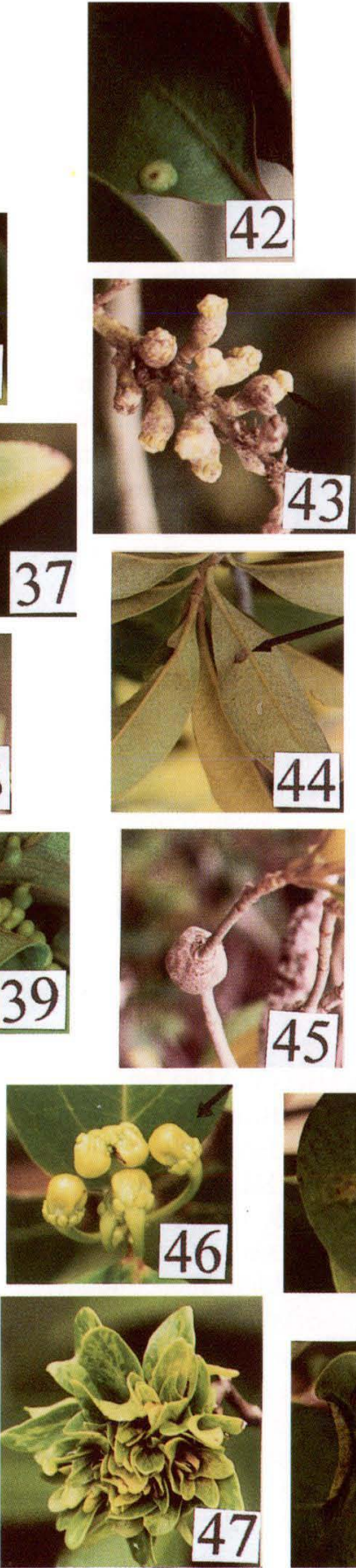
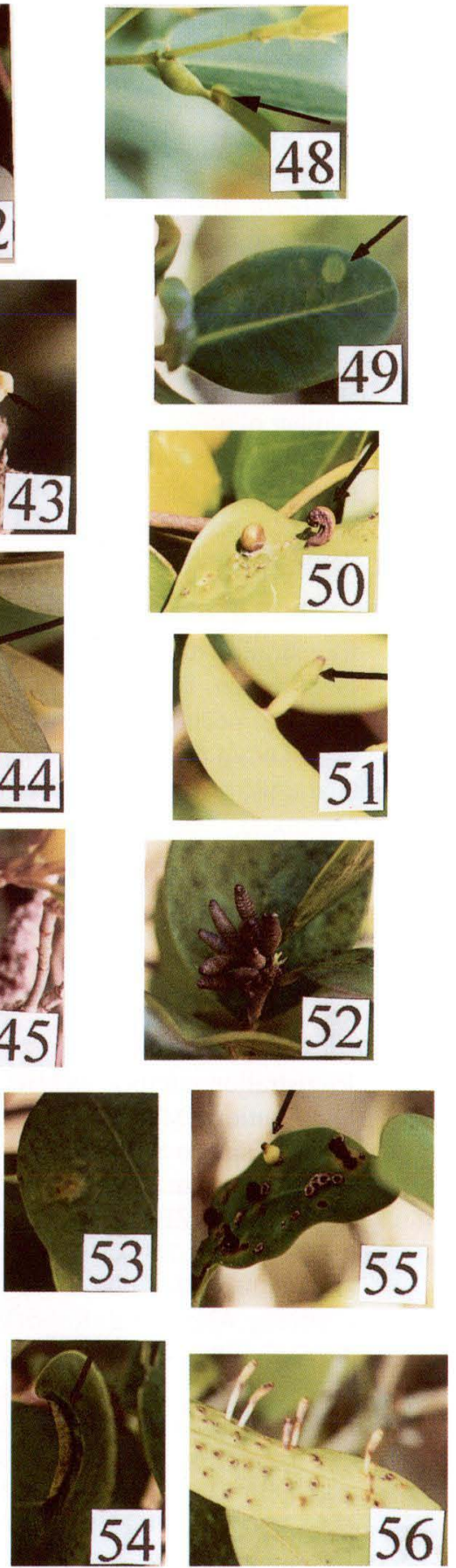
( 1 on 28.VIII.1998, 3 on 29.VIII.1998, 2 on 25.IX. 1998). Other arthropods: Hymenoptera. Periods of larva occurrence: August-February. Points of occurrence: the same ones recorded to the host plant.

\section{Loranthaceae}

\section{Psittacanthus dichrous (Mart.) Mart.}

Registration number: 178110 (A. Souza leg.)

Distribution: from Brazil (Piauí, Paraiba, Alagoas, Santa Catarina, Bahia, Minas Gerais, Espírito Santo, Rio de Janeiro, São Paulo) to Paraguay.

Points of occurrence: RBM: MLP $-3000 \mathrm{~m}$.

Gall (Fig. 42). Circular leaf gall; diameter: $0.4 \mathrm{~cm}$; glabrous, monothalamous; green. Number of galls/leaf: 1-4. Gall maker: Clinodiplosini (Cecidomyiidae). Pupation in the soil. Number of gall midge larvae/gall: 1. Locality: RBM. Material: 4 larvae (3 on 20.IV.1987, 1 on 15.IX.1998), 1 pupal exuviae (col.: 08.IX.1998, emerg.: 28.IX.1998); 2 females (col.: 08.IX.1998, emerg.: 28.IX.199). Periods of gall occurrence: April- September. Points of occurrence: RBM: CLP-3000m.

\section{Struthanthus maricensis Rizz.}

Registration number: 161002 (A. Souza leg.)

Distribution: Brazil (Rio de Janeiro).

Points of occurrence: RBM: MLP - 2000-2400m. RI: 1800, 2200m.

Gall. Ovoid midvein swelling; length: $1.0-1.5 \mathrm{~cm}$; width: $0.6 \mathrm{~cm}$; glabrous, monothalamous; green. Gall maker: Asphondylia maricensis Maia \& Couri, 1992 (Cecidomyiidae). Pupation in the gall. Number of gall midge larvae/gall: 1. Locality: RBM. Material: 6 galls (25.I.1998). Periods of gall occurrence: February, May, October. Points of occurrence: the same ones recorded to the host plant. Comments. Gall and gall maker described by MAIA et al. (1992).

\section{Malpighiaceae}

\section{Byrsonima sericea DC.}

Registration number: 195102 (V. Maia leg.)

Distribution: Martinica and Brazil (Ceará, Piauí, Pernambuco, Sergipe, Bahia, Goiás, Espírito Santo, Rio de Janeiro, Paraná).

Points of occurrence: RBM: MLP - 200-1000, 1400-2000, 2400m-3200, 3600-5600, 6000m; ZBP - 200-1000, 1400-2400, 2800, 3200, 3600-4200, 46006000m. RI: 200-400, 2800, 3400-4000m. RC: CLP - 200-600, 1200-4200m; PGP $-200,800,1000,1600-2000,2400-2600,3000,3400 \mathrm{~m}$.

Gall (Fig. 43). Ovoid closed flower; length: $1.2-1.5 \mathrm{~cm}$; medial width: 0.5-0.6 $\mathrm{cm}$; glabrous, polithalamous; green or brown. Gall maker: Asphondylia byrsonimae Maia \& Couri, 1992 (Cecidomyiidae). Pupation in the gall. Number of gall midge larvae/gall: 1-3. Localities: RBM and RC. Material: RBM: 2 females (17.X.1987), 2 males (17.X.1987), 8 galls (24.IV.1998); RC: 22 pupal exuviae (29.VIII.1998), 7 galls (29.VI.1998). Periods of gall occurrence: April-February. Points of gall occurrence: RBM: MLP - 200, 2600m; RC: CLP - 1600-1800, 2400-3200m; PGP $-400 \mathrm{~m}$. Comments. Gall and gall maker described by MAIA et al. (1992). 
Gall (Fig. 44). Circular leaf gall; diameter: $0.3 \mathrm{~cm}$; glabrous, monothalamous; green on superior surface and brown on inferior surface. Number of galls/leaf: 1-5. Gall maker: Oligotrophini (Cecidomyiidae). Pupation in the gall. Number of gall midge larvae/gall: 1. Localities: RBM, RI and RC. Material: RBM: 8 larvae (7 on III.1988, 1 on 21.VIII.1998), 1 pupal exuviae (05.VI.1998), 1 female (05.VI.1998), 1 male (05.VI.1998), 37 galls (5 on VIII.1990, 32 on 25.I. 1998); RC: 4 galls (23.V.1998). Other arthropods: parasitoid - Tetrastichinae (Eulophidae, Hymenoptera). Periods of gall occurrence: March-August, November-January. Points of gall occurrence: RBM: MLP - 200, 1600, 3600, 4000, 5200m; ZBP-2800, 4000, 4800- 5200, 5600, 6000m; RI: 400, 3400-3600m; RC: CLP - 200-600, 1200-2000, 2400, 2800-3000, 3400-4200m; PGP - 200, 800$1000,1600,2000,2400-2600,3000 \mathrm{~m}$.

Gall (Fig. 45). Ovoid stem swelling; length: $1.5 \mathrm{~cm}$; width: $0.8 \mathrm{~cm}$; glabrous, mono- or polithalamous; brown. Gall maker: Cecidomyiinae. Pupation in the gall. Number of gall midge larvae/chamber: 1. Localities: RBM, RI and RC. Material: RBM: 3 galls (2 on 12.XII.1997, 1 on 14.III.1998); RC: 1 larva (24.V.1998). Other arthropods: Hymenoptera. Periods of gall occurrence: all months of the year. Points of gall occurrence: RBM and RI: the same ones recorded by host plant: RC: CLP 200, 1400-1600, 2200-3000, 3400, 3800-4200m; PGP - 1800-2000m.

\section{Heteropteris nitida DC.}

Registration number: 38993 (J. Vidal leg.)

Distribution: Brazil (Bahia, Minas Gerais, Espírito Santo, Rio de Janeiro, São Paulo, Paraná).

Points of occurrence: RBM: MLP - 2800, 4200, 5400-6200m; ZBP 200-600, 4000, 4400, 5400-6400m. RI: 400m.

Closed flower (Fig. 46) with gall midge larvae; length: 0.6-0.7 cm; width: 0.5-0.6 cm; yellow. Gall midge associated: Clinodiplosis sp. (Cecidomyiidae). Pupation in the soil. Number of gall midge larvae/closed flower: 1-3. Locality: RBM. Material: 3 larvae (28.I.1998), 3 males (1 on 28.I.1998; 1 col.: 05.IV.1998, emerg.: 17.IV.1998; 1 col. 05.VI.1998, emerg.: 04.VII.1998), 5 galls (2 on 28.I. 1991; 3 on 05.VI.1998). Periods of damage: January, March-April, June, November-December. Points of occurrence: RBM: the same ones recorded to the host plant.

\section{Tetrapteris phlomoides (Spr.) Nied.}

Registration number: 178115 (A. Souza leg.)

Distribution: Brazil (Espírito Santo, Rio de Janeiro).

Points of occurrence: RBM: MLP - 6000m; ZBP - 6200-6400m.

Gall (Fig. 47). Rosette bud gall with small cylinders at the bottom; cylinder length: $0.4 \mathrm{~cm}$; cylinder width: $0.1 \mathrm{~cm}$; glabrous, monothalamous; green. Gall maker: Asphondyliini (Cecidomyiidae). Pupation in the gall. Number of gall midge larvae/cylinder: 1. Locality: RBM. Material: 8 pupal exuviae (30.IV.1990), 8 females (17.VII.1998), 2 males (1 on 17.VII.1988, 1 on 30.IV. 1990), 12 galls (11 on 30.IV.1990, 1 on 05.IV.1998). Periods of gall occurrence: January, April, June-July. Points of gall occurrence: MLP - 6000m; ZBP- 6200-6400m. 


\section{Melastomataceae}

\section{Miconia cinnamomifolia (DC.) Naudin.}

Registration number: 195125 (V. Maia leg.)

Distribution: Brazil (from Bahia to Santa Catarina).

Points of occurrence: RC: CMP - 200-400, 1600, 2000, 3200-3400m; PGP $-400,1400,3000 \mathrm{~m}$.

Gall (Fig. 48). Ovoid bud gall; length: $0.8-1.1 \mathrm{~cm}$; width: $0.3-0.6 \mathrm{~cm}$; glabrous, mono- or polithalamous; green. Gall maker: Epihormomyia sp. (Cecidomyiidae). Pupation in the gall. Number of gall midge larvae/chamber: 1. Locality: RC. Material: 5 larvae (1 on 29.VI.1998, 1 on 25.IX.1998, 1 on 29.I.1999, 1 on 30.I.1999, 1 on 17.III.1999), 1 pupal exuviae (31.X.1998), 4 females (31.X.1998), 2 males (1 on 31.X.1998, 1 on 28.XI.1998), 13 galls (29.VI.1998). Other arthropods: inquiline: Resseliella sp. (Cecidomyiidae) - 3 larvae on 27.II.1999; parasitoid: Hymenoptera. Periods of gall occurrence: June-March. Points of gall occurrence: CLP - 1600, 2000, 3200-3400m; PGP - 1400, 3000m.

\section{Myrsinaceae}

\section{Rapanea parvifolia (A. DC.) Mez.}

Registration number: 195186, 195187 (V. Maia leg.)

Distribution: Brazil (Bahia, Rio de Janeiro, São Paulo, Paraná, Santa Catarina, Rio Grande do Sul).

Points of occurrence: RBM: MLP - 400, 800-1000, 1400-1800, 2200-3400, 3800, 4200, 4600-5800m; PGP - 200-600, 1000-2400, 3200-6200m. RI: 400, 800, 1200-1400, 1800-2200, 2803000, 4000m. RC: CLP - 200-400, 1800-2600, 36004200m; PGP - 600, 1600-2400, 2800-3000, 3400m.

Gall (Fig. 49). Circular leaf gall; diameter: $0.4 \mathrm{~cm}$; glabrous, monothalamous; green. Number of galls/leaf: 1-5. Gall maker: Cecidomyiinae. Pupation in the gall. Number of gall midge larvae/gall: 1. Localities: RBM, RI and RC. Material: RBM: 7 larvae (1 on 28.XII.1987, 4 on 28.XII.1988, 2 on 02.XII.1989), 7 galls (2 on 02.XII.1989, 5 on 05.IV.1998); RC: 11 galls (23.V.1998). Periods of gall occurrence: January, March-April, June, December. Points of gall occurrence: RBM: MLP - 4400-4600m; ZBP - 200, 4200-4400, 5000m. RI: 800m. RC: CLP400, 1800-2600, 3600-3800, 4200m; PGP -1600, 2000-2400, 2800m.

\section{Myrtaceae}

\section{Eugenia copacabanensis Kiaersk.}

Registration number: 195279, 195280 (V. Maia leg.)

Distribution: Brazil (Bahia, Rio de Janeiro).

Points of occurrence: RBM: MLP - 800, 600, 2600-3800, 5200-5400, 5800m; PGP- 2000, 2400-3000, 3400, 5800m.

Gall (Fig. 50). Spiral leaf gall; length: $0.4 \mathrm{~cm}$; width: $0.2 \mathrm{~cm}$; glabrous, monothalamous; green or red. Number of galls/leaf: 1-33. Gall maker: Stephomyia espiralis Maia, 1993 (Cecidomyiidae). Pupation in the gall. Number of gall midge 
larvae/gall: 1. Locality: RBM. Material: RBM: 6 larvae (1 on 21.X.1997, 1 on 25.I.1998, 4 on 05.X.1998), 1 pupal exuviae (06.XII.1997), 1 pupa (25.I.1998), 59 galhas (1 on 21.X.1997, 2 on 06.XII.1997, 56 on 12. XII.1997). Other arthropods: parasitoid - Tetrastichinae, n. gen. (Eulophidae, Hymenoptera). Periods of gall occurrence: January, May-June, October-December. Points of gall occurrence: MLP - 2600, 3600-3800, 5200, 5800m. Comments. Gall and gall maker described by MAIA (1993c).

Gall (Fig. 51). Fusiform leaf gall; length: $0.9 \mathrm{~cm}$; width: $0.2 \mathrm{~cm}$; glabrous; monothalamous; green or red. Number of galls/leaf: 1-37. Gall maker: Stephomyia tetralobae Maia, 1993 (Cecidomyiidae). Pupation in the gall. Number of gall midge larvae/gall: 1. Locality: RBM. Material: 8 larvae (2 on 23.X.1992, 6 on 21.X.1997), 6 pupal exuviae (06.XII.1997), 7 females (06.XII.1997), 2 males (10.IX.1993), 60 galls (06.XII.1997). Other arthropods: parasitoids - Rileya sp. and Tetrastichinae, n. gen. (Hymenoptera). Periods of gall occurrence: January, June, September-December. Points of gall occurrence: MLP - 1600, 2600, 3800, 5400m. Comments. Gall and gall maker described by MAIA (1993c).

\section{Eugenia multiflora Camb.}

Registration number: 195294 (V. Maia leg.)

Distribution: Brazil (Rio de Janeiro, South Region).

Points of occurrence: RC: CLP - 200-600, 1200-1600, 2000-2800, 4200m; PGP - 1200-1600, 2600-3400m.

Gall (Fig. 52). Cylindrical stem gall; length: $1.1 \mathrm{~cm}$; width: $0.2 \mathrm{~cm}$; glabrous, monothalamous; brown. Gall maker: Stephomyia sp. (Cecidomyiidae). Pupation in the gall. Number of gall midge larvae/gall: 1. Locality: RC. Material: 8 larvae (2 on 27.VI.1998, 3 on 29.VI.1998, 3 on 29.VIII.1998), 3 pupal exuviae (1 on 25.IX.1998, 1 on 31.X.1998, 1 on 27.XI.1998), 4 females (1 on 31.X.1998, 3 on 27.XI.1998), 11 galls (23.V.1998). Other arthropods: inquiline - Leptothorax sp. (Formicidae, Hymenoptera); parasitoids - Eupelmidae and Platygastridae (Hymenoptera). Periods of gall occurrence: May-February. Points of gall occurrence: CLP - 200-600, 1200-1600, 2000-2400, 2800, 4200m; PGP - 1600, 3400m.

Gall (Fig. 53). Circular leaf gall; diameter: $0.4-0.5 \mathrm{~cm}$; glabrous; monothalamous; green. Number of galls/leaf: 1-14. Gall maker: Lasiopteridi (Cecidomyiidae). Pupation in the gall. Number of gall midge larvae/gall: 1. Locality: RC. Material: 2 larvae (19.XII.1998), 1 female (26.IX.1998), 2 males (27.XI.1998), 10 galls (23.V.1998). Other arthropods: parasitoid - Hymenoptera. Periods of gall occurrence: May-February. Points of gall occurrence: CLP - 200-600, 1200-1600, 2000-2400, 2800m; PGP - 1200-1600, 2200, 2600-2800, 3400m.

Gall (Fig. 54). Marginal leaf roll; length: $1.5-2.5 \mathrm{~cm}$; width: $0.2 \mathrm{~cm}$; glabrous; monothalamous; green. Number of galls/leaf: 1-2. Gall maker: Cecidomyiinae. Pupation in the gall. Number of gall midge larvae/gall: 1. Locality: RC. Material: 9 larvae (27.XI.1998), 1 pupal exuviae (23. V.1998), 2 galls (23.V.1998). Other arthropods: parasitoid - Eulophidae (Hymenoptera). Periods of gall occurrence: May-February. Points of gall occurrence: CLP - 200-600, 1200-1600, 2000-2200, 2600m; PGP - 1400, 1600, 2200-2800, 3200-3400m. 
Gall (Fig. 55). Pyriform leaf gall, wide at the base and tapered at the apex; length: $0,3-0,5 \mathrm{~cm}$; basal width: $0,3 \mathrm{~cm}$; glabrous; monothalamous; yellow. Number of galls/leaf: 1-5. Gall maker: Cecidomyiinae. Pupation in the gall. Number of gall midge larvae/gall: 1. Locality: RC. Material: 2 larvae (20.III.1999). Other arthropods: parasitoids - Eupelmidae and Eulophidae (Hymenoptera). Periods of gall occurrence: July-March. Points of gall occurrence: CLP - 200, 1600m.

Gall (Fig. 56). Claviform leaf gall; length: $0.4 \mathrm{~cm}$; basal width: $0,2 \mathrm{~cm}$; glabrous; monothalamous; green or red. Number of galls/leaf: 1-49. Gall maker: Stephomyia clavata ? (Tavares, 1920) (Cecidomyiidae). Pupation in the gall. Number of gall midge larvae/gall: 1. Locality: RC. Material: 26 pupal exuviae (25 on 26.IX.1998, 1 on 19.XII.1998), 10 females (6 on 26.IX.1998, 3 on 31.X.1998, 1 on 19.XII.1998), 2 males (26.IX.1998), 7 galls (23.V.1998). Other arthropods: parasitoids - Eulophidae, Eupelmidae and Platygastridae (Hymenoptera). Periods of gall occurrence: May, August-December. Points of gall occurrence: CLP 200-400, 1200m. Comments. TAVARES (1920) described Stephomyia clavata and its gall on an undetermined Myrtaceae. The types of this species are possibly lost and its original description is superficial. HOUARD (1933) showed a drawing of the galls. GAGNÉ (1994) transferred this species originally included in Oxasphondylia to Stephomyia, based on its gall morphology.

Gall (Fig. 57). Leaf gall, triangular; length: $0,5 \mathrm{~cm}$; basal width: $0,4 \mathrm{~cm}$; glabrous; monothalamous; yellow. Number of galls/leaf: 1-7. Gall maker: Cecidomyiinae. Pupation in the soil. Number of gall midge larvae/gall: 1. Locality: RC. Material: 7 larvae (4 on 31.X.1998, 1 on 26.II.1999 and 2 on 20.III.1999). Periods of gall occurrence: October-February. Points of gall occurrence: PC $-200,1600 \mathrm{~m}$.

\section{Eugenia rotundifolia Casar}

Registration number: 195293, 195281 (V. Maia leg.)

Distribution: Brazil (Rio de Janeiro, Bahia).

Points of occurrence: RBM: MLP - 400-2000, 2400-4600, 5000-6400m; ZBP - 200-400, 1000-6400m. RI: 200, 600-1200, 2000, 2800-4000, 4400m.

Gall (Fig. 58). Marginal leaf gall; length: $2.0-2.5 \mathrm{~cm}$; width: $0.2 \mathrm{~cm}$; glabrous; monothalamous; green. Number of galls/leaf: 1-3. Gall maker: Cecidomyiidi (Cecidomyiidae). Pupation in the gall. Number of gall midge larva/gall: 1-5. Localities: RBM and RI. Material: Cecidomyiidi - RBM: 1 male (14.VIII. 1997), 3 females (1 on 28.VI.1992, 1 on 30.XI.1992, 1 on 22.VIII.1993), 2 pupal exuviae ( 1 on 07.III.1988, 1 on 22.VIII.1993), 14 galls (3 on 05.VI.1992, 4 on 12.XII.1997, 3 on 25.I.1998, 4 on 22.III.1998); RI: 6 galls (03.IV.1998). Other

Figs 57-72. Restinga galls on Myrtaceae. (57) Triangular leaf gall on Eugenia multiflora; (58-61) on Eugenia rotundifolia: (58) marginal leaf roll; (59) circular leaf gall; (60) cylindrical stem gall; (61) modified cylindrical gall; (62-64) on Eugenia uniflora: (62) conical leaf gall; (63) circular leaf gall; (64) fruit galls; (65-67) on Myrcia ovata: (65) globular leaf gall; (66) bud gall; (67) modified bud gall; (68) flower petiole swelling on Myrcia ovata; (69-71) on Myrciaria floribunda (Myrtaceae): (69) marginal leaf roll; (70) bivalve bud gall; (71) stellate leaf gall; (72) on Neomitranthes obscura: (72a) marginal leaf roll; (72b) triangular leaf roll. 


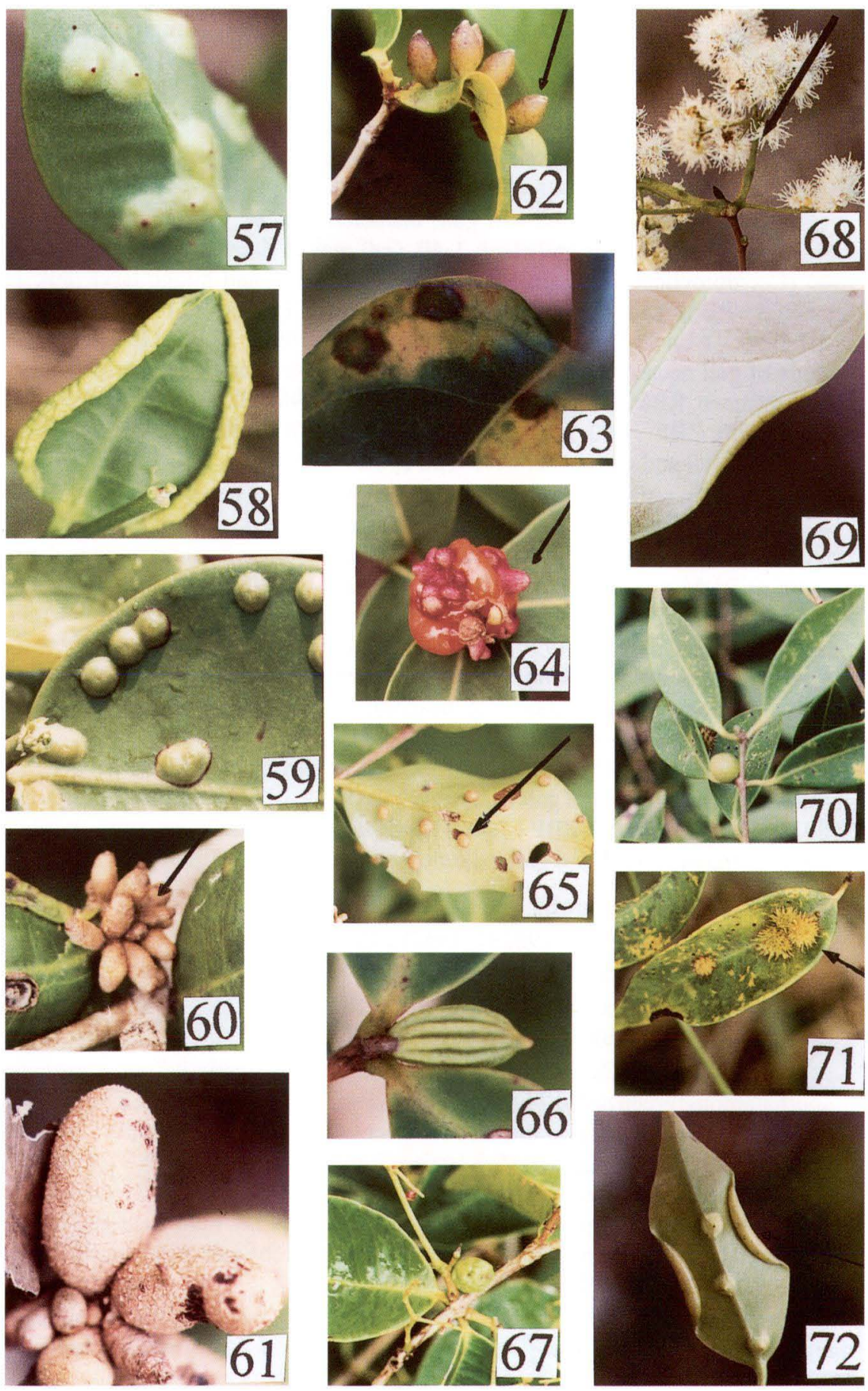


arthropods: predator - Lestodiplosis sp. (Cecidomyiidae) (1 male on 02.XII.1989), parasitoids - Tetrastichinae, n. gen. (Eulophidae) and Torymidae (Hymenoptera). Periods of gall occurrence: all months of the year. Points of gall occurrence: RBM - MLP - 400-800, 2400, 2800, 3400-3800, 4400, 5600, 6000m; ZBP - 200-400, 1000, 1400-4600, 5000-6000m; RI: 200-800, 1200-1400, 2800, 3200-3600m. Comments. Gall described by MAIA (1995b).

Gall (Fig. 59). Circular leaf gall; diameter: $0.6 \mathrm{~cm}$; glabrous; monothalamous; yellow. Number of galls/leaf: 1-40. Gall maker: Dasineura globosa Maia, 1995 (Cecidomyiidae). Pupation in the gall. Number of gall midge larva/gall: 1. Localities: RBM and RI. Material: RBM: 43 galls (11 on 30.IV.1990, 18 on 22.III.1998, 14 on 03.IV.1998); RI: 1 female (04.IX.1998), 17 galls (03.IV.1998). Periods of gall occurrence: January, March-July, September-December. Points of gall occurrence: RBM: MLP - 600-800, 1400, 1800, 2400, 2800, 3400-3800, 4200, 6000, 6400m; ZBP - 400, 1000, 1600, 2000-6000m. RI: 200-1200, 2800-4000m. Comments. Gall and gall maker described by MAIA (1995b).

Gall (Fig. 60). Cylindrical stem gall; length: $1.5-1.8 \mathrm{~cm}$; width: 0.4-0.5 cm; glabrous; monothalamous; brown. Gall maker: Stephomyia rotundifoliorum Maia, 1993 (Cecidomyiidae). Pupation in the gall. Number of gall midge larva/gall: 1. Localities: RBM and RI. Material: RBM: 6 larvae (14.VIII.1997), 37 galls (13 on 05.VI.1992, 3 on 22.XI.1997, 21 on 17.IV.1998), 7 galls modified by phytophagous wasps; RI: 89 galls (03.IV.1998). Other arthropods: parasitoids (Hymenoptera) Rileya sp. (Eurytomidae), Donquickeia n. sp. (Braconidae), Eupelmidae and Torymidae; predators - Novohorus sp. (Pseudoscorpiones: Olpiidae); inquilines-Hymenoptera. Other material: galls modified by phytophagous wasps (Fig. 61)-RBM: 7 galls (22.XI.1997); RI: 89 galls (03.IV.1998). Periods of gall occurrence: all months of the year. Points of gall occurrence: RBM-MLP -600, 1200, 1800, 2400, 2800, 3400, 3800, 6400m; ZBP - 200, 1400-1800, 2600, 5200m; RI: 200-400, 800, $1200 \mathrm{~m}$. Comments. Gall and gall maker described by MAIA (1993c).

\section{Eugenia uniflora Linnaeus}

Registration number: 195291, 195292 (V. Maia leg.)

Distribution: Brazil (Southeast and South regions), Uruguay and Argentina.

Points of occurrence: RBM: MLP-600, 1000, 1800, 4000-4400, 4800-5200, 5600-6400m; ZBP - 2600, 5200, 6200m. RI: 1600m. RC: $200 \mathrm{~m}$.

Gall (Fig. 62). Conical leaf gall; length: $0.6 \mathrm{~cm}$; width: $0.2 \mathrm{~cm}$; glabrous; monothalamous; green or red. Number of galls/leaf: 1-36. Gall maker: Cecidomyiidi (Cecidomyiidae). Pupation in the soil. Number of gall midge larva/gall: 1. Localities: RBM and RI. Material: RBM: 3 larvae (2 on 12.X.1987, 1 on 06.VI.1992), 25 galls (03.VII.1998). Other arthropods: parasitoids - Eulophidae: Chrysonotomyia sp. and Tetrastichinae n. gen.; Platygastridae (Hymenoptera). Periods of gall occurrence: January, April, June, October-December. Points of gall occurrence: RBM: MLP-3600-4200, 5200, 6000, 6200m; RI: 1600m.

Gall (Fig. 63). Circular leaf gall; diameter: $0.25 \mathrm{~cm}$; glabrous; monothalamous; green or red. Number of galls/leaf: 1-10. Gall maker: Neolasioptera engeniae Maia, 1993 (Cecidomyiidae). Pupation in the gall. Number of gall midge larva/gall: 
1. Locality: RBM. Material: RBM: 29 galls (4 on 30.IV.1990, 25 on 05.IV.1998). Periods of gall occurrence: January, March-April, June, November-December. Points of gall occurrence: MLP- 3600-4200, 5600, 6000m; ZBP - 5200, 6200m. Comments: Gall and gall maker described by MAIA (1993a).

Gall (Fig. 64). Fruit gall; triangular; length: $0.3 \mathrm{~cm}$; width: $0.2 \mathrm{~cm}$; glabrous; monothalamous; red. Number of galls/fruit: 1-14. Gall maker: Cecidomyiinae. Number of gall midge larva/gall: 1. Locality: RBM. Material: 6 galls (17.VII.1997). Other arthropods: parasitoid - Eurytomidae (Hymenoptera) Periods of gall occurrence: July. Points of gall occurrence: MLP $-4800 \mathrm{~m}$.

\section{Myrcia ovata Camb.}

Registration number: 195282 (V. Maia leg.)

Distribution: Brazil (Rio Grande do Norte, Rio de Janeiro).

Points of occurrence: RBM: ZBP - 600m. RC: CLP - 400, 1200-2000, 2600m; PGP - 200, 600-800, 1400-1600, $2200 \mathrm{~m}$.

Gall (Fig. 65). Globular leaf gall; diameter: $0.1 \mathrm{~cm}$; glabrous; monothalamous; yellow. Number of galls/leaf: 1-8. Gall maker: Cecidomyiinae. Pupation in the gall. Number of gall midge larva/gall: 1. Localities: RBM and RC. Material: RC: 1 female (19.XII.1998), 5 galls (25.IV.1999). Other arthropods: inquiline -Tetrastichinae (Hymenoptera: Eulophidae). Periods of gall occurrence: April-February. Points of gall occurrence: RBM: the same one recorded to the host plant; RC: CLP - 400-600, 1200-2000m; PGP - 400-800, 2200m.

Gall (Fig. 66). Bud gall; ovoid with longitudinal ridges extending from base to apex; length: $1.0 \mathrm{~cm}$; width: $0.2-0.3 \mathrm{~cm}$; glabrous; monothalamous; yellow. Gall maker: Myrciamyia maricaensis Maia, 1995 (Cecidomyiidae). Pupation in the gall. Number of gall midge larva/gall: 1. Localities: RBM and RC. Material: RBM: 4 galls (2 on 04.VIII.1992, 2 on 25.IV.1998); RC: 2 galls (28.VI.1998). Other arthropods: inquiline - Aprostocetus sp. (Hymenoptera: Eulophidae). Other material: galls modified by phytophagous wasps (Fig. 67) - RBM: 3 galls (25.IV.1998); RC: 2 galls (28.VI.1998). Periods of gall occurrence: April, June, August. Points of gall occurrence: RBM: ZBP- 600m; RC: CLP - 200, 1400, 2000m; PGP - 1600m. Comments. Gall and gall maker described by MAIA (1995a).

Gall (Fig. 68). Flower peduncle swelling; ovoid; length: $1.3 \mathrm{~cm}$; width: $0.2 \mathrm{~cm}$; glabrous; monothalamous; green. Gall maker: Cecidomyiinae. Pupation in the gall. Number of gall midge larva/gall: 1. Localities: RBM and RC. Material: RBM: 1 larva (22.V.1992), 3 galls (16.V.1998); RC: 1 larva (24.VII.1998), 1 gall (23.V.1998). Periods of gall occurrence: April-August. Points of gall occurrence: RBM: ZBP- 600m; RC: CLP - 400, 1200-1600, 2000m; PGP - 2200m.

\section{Myrciaria floribunda (West ex Willdenow) Berg.}

Registration number: 195283, 195284 (V. Maia leg.)

Distribution: Neotropical (from Caribe to South of Brazil).

Points of occurrence: RBM: MLP - 200-800, 1200-6000, 6400m; ZBP 600-800m. RC: CLP-400, 1200-2000, 2600m; PGP-200-2000, 2400, 4000-4200, 5000, 5600-6000 m. RI: 1000m. RC: CLP-200, 600, 1200, 2600m; PGP-600-800, 1600-1800, $2200 \mathrm{~m}$. 
Gall (Fig. 69). Marginal leaf roll; length: $0.6 \mathrm{~cm}$; width: $0.1 \mathrm{~cm}$; glabrous; monothalamous; green. Number of galls/leaf: 1-3. Gall maker: Dasineura myrciariae Maia, 1993 (Cecidomyiidae). Pupation in the gall. Number of gall midge larva/gall: 1. Localities: RBM, RI and RC. Material: RBM: 9 larvae (2 on I.1993, 1 on 09.III.1993, 5 on 10.X.1997, 1 on 08.IX.1998), 2 pupal exuviae (10.X.1997), 1 pupa(10.X.1997), 1 female (10.X.1997), 8 galls (4 on 22.VI.1992, 4 on 25.I.1998); RI: 5 galls (03.IV.1998); RC: 1 larva (27.XI.1998), 9 galls (24.V.1998). Other arthropods: predator - Lestodiplosis sp. (Cecidomyiidae); parasitoid - Proacrias sp. (Hymenoptera: Eulophidae). Periods of gall occurrence: January, March, MayFebruary. Points of gall occurrence: RBM: MLP-200-600, 1600, 2800, 3400, 5400, 6000 m; ZBP- 200-400, 1000-2400, 4000-4200, 5000, 5600-6000m; RI: $1000 \mathrm{~m}$; $\mathrm{RC}$ : CLP - the same ones recorded to the host plant; PGP $-600,1600,2200 \mathrm{~m}$. Comments. Gall and gall maker described by MAIA (1995b).

Gall (Fig. 70). Bivalve bud gall; ovoid; length: $0.7 \mathrm{~cm}$; width: $0.5 \mathrm{~cm}$; glabrous; monothalamous; green or yellow. Gall maker: Myrciariamyia bivalva Maia, 1994 (Cecidomyiidae). Pupation in the gall. Number of gall midge larvae/gall: 1. Localities: RBM and RC. Material: RBM: 12 galls (2 on 19.VII.1987, 1 on 08.VI.1992, 1 on 06.VII.1992, 8 on 29.VIII.1993); RC: 1 pupal exuviae (24.VIII. 1998), 1 pupa (24.VIII.1998). Periods of gall occurrence: June-August. Points of gall occurrence: RBM: MLP - 200-600, 1400-1800, 2400, 3000-3200, 4600-4800 m; ZBP- $600 \mathrm{~m}$; RC: CLP-1200m; PGP - 2200m. Comments. Gall and gall maker described by MAIA (1994).

Gall (Fig. 71). Stellate leaf gall (diameter: $0.4 \mathrm{~cm}$ ) with a small cylinder in the middle; glabrous; monothalamous; green or yellow. Number of galls/leaf: 1-5. Gall maker: Cecidomyiinae. Pupation in the gall. Number of gall midge larva/gall: 1. Locality: RC. Material: 1 larva (24.V.1998), 6 galls (24.V.1998). Other arthropods: parasitoid - Hymenoptera. Periods of gall occurrence: May-February. Points of gall occurrence: RC: the same ones recorded to the host plant.

\section{Neomitranthes obscura (DC.) N. J. E. Silveira}

Registration number: 195285, 195286 (V. Maia leg.)

Distribution: Brazil (Rio de Janeiro, Espírito Santo).

Points of occurrence: RBM: MLP-400-1400, 1800, 2200-3400, 3800-4000, 4400-4600, 5000-5600m; ZBP - 2000-2400, 2800-4600, 5000, 5800-6200m. RI: 400, 1800, 2800m. RC: CLP - 200, 600, 1200, 1600, 2000-2600, 4200m; PGP 200-600, 1000-1200, 2200, 2600-2800m. RI: 400, 1800, 2800m. RC: CLP - 200, $600,1200,1600,2000-2600,4200$ m; PGP - 200-600, 1000-1200, 2200, 2600$2800 \mathrm{~m}$.

Gall (Fig. 72). Marginal leaf roll; length: $1.0 \mathrm{~cm}$; width: $0.5 \mathrm{~cm}$; glabrous; monothalamous; green. Number of galls/leaf: 1-4. Gall midges: Dasineura tavaresi Maia, 1993 (Cecidomyiidae) and Clinodiplosis sp. (Cecidomyiidae). Pupation in the gall. Number of gall midge larva/gall: Dasineura tavaresi: 1; Clinodiplosis sp.: 2-5. Localities: RBM, RI and RC. Material: RBM: 8 galls (5 on 27.VIII.1992, 3 on 05.IV.1998); RC: 6 galls (23.V.1998); Clinodiplosis sp. - RI: 7 larvae (10.VII. 1998), RC: 2 larvae (27.II.1999). Other arthropods: free living inquiline - Stenoma 
annosa (Lepidoptera). Periods of gall occurrence: May-February. Points of gall occurrence: RBM: MLP - 400-600, 3400, 4400-4600 m;ZBP- 200-600, 1000-1800, 2200-2400, 3000-4600, 5000, 5800-6200m; RI: 1800, 2800m; RC: CLP-200-600, 1200-2000, 2400-2600, 4200m; PGP - 200-600, 1000-1200, 1600, 2200, 2600$2800 \mathrm{~m}$. Comments. Gall and gall maker described by MAIA (1993c).

Gall (Fig. 72). Triangular leaf gall; length: $0.2 \mathrm{~cm}$; basal width: $0.3 \mathrm{~cm}$; glabrous; monothalamous; red or green. Number of galls/leaf: 1-5. Gall maker: Cecidomyiinae. Pupation in the soil. Number of gall midge larva/gall: 1. Localities: RBM and RC. Material: RBM: 14 galls (8 on 27.VIII.1992, 2 on 28.VIII.1992, 4 on 17.IV.1998). Periods of gall occurrence: April, November-December. Points of gall occurrence: RBM: MLP - 3800; RC: PGP - 1000, 2200m.

Gall (Fig. 73). Pine-like bud gall; length: $2.5 \mathrm{~cm}$; basal width: $0.8 \mathrm{~cm}$; glabrous; without internal chamber; green. Gall maker: Neomitranthella robusta Maia, 1995 (Cecidomyiidae). Pupation in the gall. Number of gall midge larva/gall: 4-21. Localities: RBM and RC. Material: RBM: 5 larvae (3 on 20.VII.1990, 3 on 23.IX.1992), 1 male (22.VII.1992), 10 galls (8 on 11.VIII.1990, 2 on 17.IV.1998); RC: 10 galls (23.V.1998). Other arthropods: parasitoid -Tetrastichinae, n. gen. (Eulophidae, Hymenoptera). Periods of gall occurrence: March-February. Points of gall occurrence: RBM: MLP - 400, 2800, 4400-4600m; ZBP - 600, 1000, 1400 $1600,2200,3600-3800,4400-4600 \mathrm{~m}$; RC: CLP - 200-600, 1200-1600, 2000, 2400-2600, 4000-4200m; PGP - 200-400, 800-1200, 1600, 2200, 2600-3000, $3400 \mathrm{~m}$. Comments. Gall and gall maker described by MAIA (1995a).

Gall (Fig. 74). Conical leaf roll; length: $0.8 \mathrm{~cm}$; width: 0.1-0.2 cm; glabrous; monothalamous; green or red. Number of galls/leaf: 1-7. Gall maker: Stephomyia mina Maia, 1993 (Cecidomyiidae). Pupation in the gall. Number of gall midge larva/gall: 1. Localities: RBM and RC. Material: RBM: 3 galls (2 on 15.I.1989, 1 on 28.XI.1993); RC: 7 galls (24.V.1998). Other arthropods: parasitoid - Hymenoptera. Periods of gall occurrence: January, June-November. Points of gall occurrence: RBM: MLP - 2200m; RC: CLP - 1200, 2000, 4200m; PGP - 800, 2200m. Comments. Gall and gall maker described by MAIA (1993c).

\section{Nyctaginaceae}

\section{Guapira opposita (Vell.) Reitz.}

Registration number: 195297 (V. Maia leg.)

Distribution: Brazil (nearly all states).

Points of occurrence: RBM: MLP - 200-3800, 4400-4600, 5000-5600, 6000-6400m; ZBP-200-6600m. RI: 200-1800, 2200, 2800, 3200-3800m. RC: CLP $-200,1200,3000,3400-3600 \mathrm{~m}$.

Gall (Fig. 75). Circular leaf gall; diameter: 0,3 cm; glabrous; monothalamous; green. Number of galls/leaf: 1-20. Gall maker: Bruggmannia elongata Maia \& Couri, 1993 (Cecidomyiidae). Pupation in the gall. Number of gall midge larva/gall: 1. Localities: RBM, RI and RC. Material: RBM: 9 larvae (28.II.1996), 30 galls (5 on 30.IV.1990, 25 on 05.IV.1998); RI: 1 pupal exuviae (10.VII.1998); RC: 15 pupal exuviae (26.IX.1998), 1 pupa (29.VI.1998), 8 female (26.IX.1998), 
7 males (10.VII.1998). Other arthropods: parasitoids - Rileya sp. and Eurytoma sp. (Eurytomidae), Platygaster sp. (Platygastridae), Galeopsomyia sp., Chrysotomyia sp., Cirrospilus sp. and Pentastichus sp. (Eulophidae). Periods of gall occurrence: January-December. Points of gall occurrence: RBM: MLP - 200, 600-800, 1400, 1800, 2000, 3000, 3400-3800, 5200-5400, 6200m; ZBP- 200-1800, 2200-6600m; RI: the same ones recorded to the host plant; RC: CLP - 200-400, 1200, 3000, 3400-3600m; PGP - 1000, 1400, 1800, 2000-2200, 3200m. Comments. Gall and gall maker described by MAIA \& COURI (1993).

Gall (Fig. 76). Rosette leaf with tubular galls in the base; tube length: $0.4 \mathrm{~cm}$; tube width: $0.1 \mathrm{~cm}$; glabrous; monothalamous; green. Gall maker: Pisphondylia braziliensis Maia \& Couri, 1992 (Cecidomyiidae). Pupation in the gall. Number of gall midge larva/tube: 1. Locality: RBM. Material: 1 male (08.IX.1998). Periods of gall occurrence: August-September. Points of gall occurrence: RBM: MLP 4000m. Comments. Gall and gall maker described by CouRI \& MAIA (1992).

Gall (Fig. 77). Stem swelling; ovoid; length: $0.8 \mathrm{~cm}$; width: $0.4 \mathrm{~cm}$; glabrous; polithalamous; brown. Gall maker: Proasphondylia guapirae Maia \& Couri, 1993 (Cecidomyiidae). Pupation in the gall. Number of gall midge larva/chamber: 1 . Localities: RBM, RI and RC. Material: RBM: 5 larvae (10.X.1997), 4 pupal exuviae (2 on 21.X.1997, 2 on 21.XI,1997), 1 pupa (21.X.1997), 1 female (10.X.1997), 2 males (1 on 10.X.1997, 1 on 21.XI.1998), 6 galls (1 on 30.IV.1990, 4 on 05.IV.1998, 1 on 25.IV.1998); RC: 1 gall (28.VI.1998). Periods of gall occurrence: April-June, October-January. Points of gall occurrence: RBM: MLP - 200-800, 1600-1800, $2000 \mathrm{~m}$; ZBP- $3400 \mathrm{~m}$; RI: the same ones recorded to the host plant; RC: CLP $200-400,1200,3000,3400-3600 \mathrm{~m}$. Comments. Gall and gall maker described by MAIA \& COURI (1993).

Gall (Fig. 78). Triangular leaf gall (projecting on both surfaces); length: 0.6 $\mathrm{cm}$; basal width: $0.4 \mathrm{~cm}$; glabrous; monothalamous; green. Number of galls/leaf: 1-7. Gall maker: Bruggmannia sp. (Cecidomyiidae). Pupation in the gall. Number of gall midge larva/gall: 1. Localities: RBM, RI and RC. Material: RBM: 1 larva (20.IV.1988), I pupal exuviae (05.X.1998), 3 females (1 on 12.IX.1987, 1 on 17.XII.1987, 1 on X.1988), 2 males (1 on 17.XII.1987, 1 on 05.X.1998), 13 galls (01 on 30.IV.1990, 12 on 22.I.1998); RI: 1 larva (02.X.1998), 1 pupal exuviae (10.VII.1998); 1 female (10.VII.1998). Other arthropods: parasitoids - Rileya sp. and Eurytoma sp. (Eurytomidae) and Galeopsomyia sp. (Eulophidae). Periods of gall occurrence: January-December. Points of gall occurrence: RBM: MLP - 1800, 3800, 5400m; ZBP- 200-400, 800, 2600, 3000-3200m; RI: 1800m; RC: CLP $1200 \mathrm{~m}$. Comments. Gall described by MAIA \& MONTEIRO (1999).

Figs 73-87. Restinga galls. (73-74) on Myrtaceae, Neomitranthes obscura: (73) pine-like bud gall; (74) conical leaf roll; (75-79) on Guapira opposita (Nyctaginaceae): (75) circular leaf gall; (76) rosette bud gall; (77) stem swelling; (78) triangular leaf gall; (79) globular leaf gall; (80) barrel-shaped leaf gall on Ouratea cuspidata (Ochnaceae); (81) stem swelling on Ximenia maericana (Olacaceae); (82) rolled young leaf on Passiflora mucronata (Passifloraceae); (83-84) on Rubiaceae: (83) inflorescence gall on Borreria verticillata; (84) inflorescence gall on Diodia gymnocephala; (85-87) on Paullinia weinmanniaefolia (Sapindaceae): (85) rolled young leaf; (86a) conical leaf gall; (86b) modified conical leaf gall; (87) tendril swelling. 

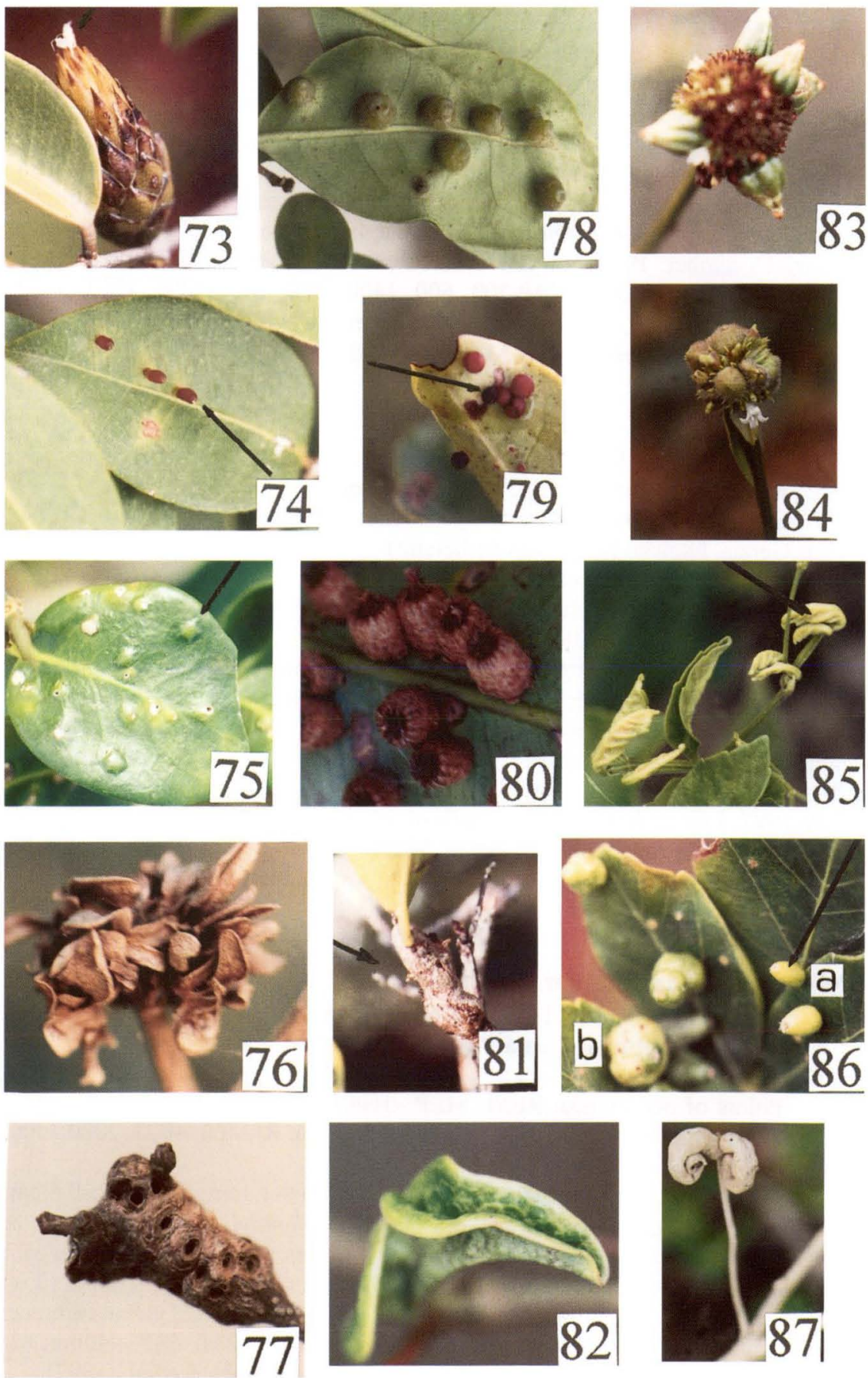
Gall (Fig. 79). Globular leaf gall; diameter: $0.2 \mathrm{~cm}$; hairy; monothalamous; red or yellow. Number of galls/leaf: 1-21. Gall maker: Bruggmannia robusta Maia \& Couri, 1993 (Cecidomyiidae). Pupation in the gall. Number of gall midge larva/gall: 1. Localities: RBM, RI and RC. Material: RBM: 4 pupal exuviae (21.IX.1992), 4 males (21.IX.1992), 6 galls (30.IV.1990); RI: 4 pupal exuviae (12.VI.1998), 4 males (12.VI.1998), 5 galls (12.VI.1998). Other arthropods: parasitoids (Hymenoptera) - Rileya sp. (Eurytomidae), Platygaster sp. (Platygastridae), Galeopsomyia sp. and Chrysotomyia sp. (Eulophidae). Periods of gall occurrence: January-December. Points of gall occurrence: RBM: MLP - 600, 1400, 1800, 3400-3600, 5200-5400 m; ZBP-200, 600, 1400-1600, 2400-3200, 4000, 44004800, 5200m; RI: 800, 1200, 1800, 2200, 3200m; RC: CLP - 2600m. Comments. Gall and gall maker described by MAIA \& COURI (1993).

\section{Ochnaceae}

\section{Ouratea cuspidata (St. Hil.) Engl.}

Registration number: 195300 (V. Maia leg.)

Distribution: Brazil (Ceará, Rio Grande do Norte, Bahia, Mato Grosso, Minas Gerais, Espírito Santo, Rio de Janeiro).

Points of occurrence: RBM: MLP - 200, 800, 1200-1600, 3600m; ZBP 200-1400m, 1800-2000, 2400. RI: 200-800, 1200-1400, 1800-2200, 2800, 32003600m. RC: CLP - 3400, 3800m; PGP - 2400m.

Gall (Fig. 80). Leaf gall; barrel-like with a pointed projection at the apex; length: $0.5 \mathrm{~cm}$; basal width: $0.3 \mathrm{~cm}$; glabrous; monothalamous; brown. Number of galls/leaf: 1-88. Gall maker: Contarinia sp. (Cecidomyiidae). Pupation in the gall. Number of gall midge larva/gall: 1. Locality: RBM. Material: RBM: 1 pupal exuviae (25.X.1998), 3 females ( 2 on 13.IV.1987, 1 on 26.VIII.1989), 2 males ( 1 on 13.IV.1987, 1 on 23.XII.1998), 145 galls (4 on 25.VIII.1989, 9 on 05.VI.1992, 132 on III.1998). Periods of gall occurrence: March-August. Points of gall occurrence: RBM: ZBP- $600 \mathrm{~m}$.

\section{Olacaceae}

\section{Ximenia americana L. var. americana}

Registration number: 145929 (J. G. da Silva leg.)

Distribution: Brazil (Ceará, Pernambuco, Bahia, Mato Grosso, Rio de Janeiro, São Paulo).

Points of occurrence: RBM: MLP -1600-1800, 2400, 3000, 3800, 4400, 6000m; ZBP - 1600m, 2400-3200, 4000-4600, 5200. RI: 400, 1000, 2000,3000, $4400 \mathrm{~m}$.

Gall (Fig. 81). Ovoid stem swelling; length: 0.9-1.1 cm; width: 0.5-0.6 cm; glabrous; monothalamous; brown. Gall maker: Asphondylia communis Maia \& Couri, 1993 (Cecidomyiidae). Pupation in the gall. Number of gall midge larva/gall: 1. Localities: RBM and RI. Material: RBM: 4 larvae (10.XI.1997), 3 males (2 on 27.IX.1 1989, 1 on 29.IX.1989); RI: 1 gall (03.IV.1998). Periods of gall occurrence: April, September-November. Points of gall occurrence: RBM: ZBP- $4400 \mathrm{~m}$; RI: 400, 1000m. Comments. Gall and gall maker described in MAIA et. al. (1992). 


\section{Passifloraceae}

\section{Passiflora mucronata Lam.}

Registration number: 195601 (V. Maia leg.)

Distribution: Brazil (Bahia, Espírito Santo, Rio de Janeiro).

Points of occurrence: RC: CLP - 200-400, 1200-2000, 2400, 4000m; PGP -200-600, 1000-1800, 2200, 3000m.

Gall (Fig. 82). Rolled young leaf; length: $1.2 \mathrm{~cm}$; width: $0.4 \mathrm{~cm}$; glabrous; monothalamous; green. Gall maker: Clinodiplosis sp. (Cecidomyiidae). Pupation in the soil. Number of gall midge larvae/gall: 1-3. Locality: RC. Material: RBM: 1 larva (31.X.1998), 1 female (col.: 30.I.1999; emerg.: 23.III.1999), 2 males (col.: 30.I.1999, emerg.: 23.III.1999). Periods of gall occurrence: October-January. Points of gall occurrence: RC: CLP - 400, 1200-1600, 2000, 2400, 4000m; PGP 1000-1200, 1600-1800, 2200,3000m.

\section{Piperaceae}

\section{Piper divaricatum Meyer}

Registration number: 195600 (V. Maia leg.)

Distribution: South America. In Brazil: Amazonas, Amapá, Ceará, Pernambuco, Bahia, Mato Grosso, Minas Gerais, Espírito Santo, Rio de Janeiro.

Points of occurrence: RC: PGP - 1400, 2200m.

Free living gall midge larvae in the inflorescence. Locality: RC. Material: 2 larvae (27.II.1999), 2 attacked inflorescence (27.II.1999). Periods of gall midge attack: February. Points of gall occurrence: PGP - 1400, 2200m.

\section{Rubiaceae}

\section{Borreria verticillata (L.) Meyer}

Registration number: 195215 (V. Maia leg.)

Distribution: from Guyana and Venezuela to Uruguay.

Points of occurrence: RBM: MLP - 200-600, 1400, 2000-2200, 3000-3600, 4000, 5000, 5800, 6200-6400m; ZBP - 400, 800-1600, 2000-2200, 4400, 54005600m. RI: 200-600, 1000-1800, 2800-3600m. RC: CLP - 200-400, 1000-1600, 2000, 2400, 2800, 3000-4200; PGP - 1000-3200m.

Gall (Fig. 83). Inflorescence gall, fusiform; length: $1.0 \mathrm{~cm}$; basal width: 0.3 cm; glabrous; monothalamous; green. Gall maker: Asphondylia borreriae Rübsaamen, 1905 (Cecidomyiidae). Pupation in the gall. Number of gall midge larvae/gall: 1 . Localities: RBM and RC. Material: RBM: 13 larvae (8 on 12.VI.1997, 5 on 06.XII. 1997), 3 pupal exuviae (2 on 17.X.1987, 1 on 12.VI.1997), 1 female (12.VI.1997), 12 galls (3 on 17.X.1987, 9 on 25.IV.1998); RC: 2 exuviae (23.V. 1998), 1 pupa (23.V.1998), 1 female (23.V.1998), 17 galls (23.V.1998). Other arthropods: parasitoids (Hymenoptera): Rileya sp. (Eurytomidae), Horismenus sp. (Eulophidae). Periods of gall occurrence: April-January. Points of gall occurrence: RBM: MLP - 200, 600, $3600 \mathrm{~m}$; RC: CLP - the same ones recorded to the host plant; PGP - 1400, 2200, 2600, $3200 \mathrm{~m}$. Comments. Gall and gall maker described by RÜBSAAMEN (1905). MAIA \& COURI (in MAIA et al. 1992) described the male, female and larva. 


\section{Diodia gymnocephala (DC.) K. Schum}

Registration number: 195598, 195599 (V. Maia leg.)

Distribution: Guyanas, Venezuela, Paraguay, Brazil.

Points of occurrence: RC: PGP - 1400, 2200, 3200m.

Gall (Fig. 84). Inflorescence gall, fusiform; length: $0.7 \mathrm{~cm}$; basal width: 0.5 $\mathrm{cm}$; glabrous; monothalamous; green. Gall maker: Clinodiplosis sp. (Cecidomyiidae). Pupation in the gall. Number of gall midge larvae/gall: 1. Locality: RC. Material: 8 larvae ( 1 on 28.VIII.1998, 7 on 26.IX.1998), 27 pupal exuviae (3 on 28.VIII.1998, 1 on 28.IX.1998, 23 on 30.I.1999), 9 females (3 on 28.VIII.1998, 3 on 25.IX.1998, 1 on 26.IX.1998, 2 on 31.X.1998), 14 males (7 on 28.VIII.1998, 2 on 25.IX.1998, 2 on 26.IX.1998, 1 on 31.X.1998, 2 on 28.XI.1998). Other arthropods: parasitoids - Platygastridae and Torymidae (Hymenoptera). Periods of gall occurrence: August-February. Points of gall occurrence: the same ones recorded to the host plant.

\section{Sapindaceae}

\section{Paullinia weinmanniaefolia Mart.}

Registration number: 195225 (V. Maia leg.)

Distribution: Brazil (Bahia, Espírito Santo, Rio de Janeiro).

Points of occurrence: RBM: MLP - 200-2600, 3000, 3400-4400, 50005800m; ZBP - 200-2800, 3400-6200m. RI: 200-1400, 1800, 2800-3400, 4000m. RC: CLP - 200-600, 1000-2800, 4000-4200m; PGP - 200, 800, 1400-1600, 2600-2800m.

Gall (Fig. 85). Rolled young leaf; length: $0.7 \mathrm{~cm}$; width: $0.1-0.2 \mathrm{~cm}$; glabrous; monothalamous; green or yellow. Gall maker: Cecidomyiinae. Pupation in the soil. Number of gall midge larvae/gall: 1. Localities: RBM, RI and RC. Material: RBM: 5 galls (28.I.1991); RC: 3 galls (23.X.1998). Other arthropods: inquiline - Clinodiplosis sp. (Cecidomyiidae), pupation in the soil; RBM: 9 larvae (4 on 28.IX.1991, 5 on 01.IX.1997), 5 females (col.: 05.VI.1998, emerg.: 16.VI. 1998, 10.VII.1998), 1 male (col.: 05.VI.1998, emerg.: 10.VII.1998); RC: 4 female (2 col. 29. VIII.1998, emerg.: 26.IX.1998, 03.X.1998; 2 col. 31.X.1998, emerg.: 09. IX.1998, 21.XI.1998), 9 males (col. 29.VIII.1998, emerg.: 2 on 24.IX.1998, 1 on 01.X.1998, 3 on 03.X.1998, 1 on 07.X.1998, 1 on 09.X.1998, 1 on 22.IX.1998); predator - Lestodiplosis sp. (Cecidomyiidae), 1 larva (21.IX.1998). Periods of gall occurrence: May-January. Points of gall occurrence: RBM: MLP - 200m; ZBP600m; RI: 1800, 2800, 3200-3400m; RC: CLP - 200-600, 1000-1600, 2000, 2400, $4200 \mathrm{~m}$. Comments. Gall described by GAGNÉ (1994).

Gall (Fig. 86a). Conical leaf; length: $0.6 \mathrm{~cm}$; width: $0.2 \mathrm{~cm}$; glabrous; monothalamous; green or yellow. Number of galls/leaf: 1-4. Gall maker: Oligotrophini n. gen. and n. sp., near Ficiomyia felt, 1922 (Cecidomyiidae). Pupation in the gall. Number of gall midge larvae/gall: 1. Localities: RBM, RI and RC. Material: RBM: 1 larva (17.VII.1992), 2 pupal exuviae (1 on 24.X.1987, 1 on II.1998), 4 females (2 on X.1987, 1 on VIII.1988, 1 on 17.VII.1992), 1 male (24.X.1987), 8 
galls (5 on 17.VII.1992, 2 on 20.VII.1995, 1 on 17.IV.1998); RI: 7 galls (22. XI.1997). Other arthropods: inquiline (Hymenoptera) - Eulophidae; parasitoids (Hymenoptera) - Eurytomidae, Platygastridae and Dimeromicrus cecidomyiae (Torymidae). Other material: galls modified by the inquiline (Fig. 86b) - RC: 2 galls modified by the inquiline (29.VI.1998). Periods of gall occurrence: April-February. Points of gall occurrence: RBM: ZBP-5800m; RI: 200-800m; RC: CLP-200-400, 1200-2800, 4000- 4200m; PGP - 200, 1400-1600, 2600m.

Gall (Fig. 87). Tendril swelling; length: 2.0-2.6 cm; width: $0.3-0.4 \mathrm{~cm}$; glabrous; monothalamous; brown. Gall maker: Cecidomyiinae. Pupation in the gall. Number of gall midge larvae/gall: 1. Locality: RC. Material: 1 pupal exuviae (26.IX.1998), 4 galls (29.VI.1998). Periods of gall occurrence: June, September. Points of gall occurrence: CLP - 1800, 2200, 2800, 4200m.

\section{Sapotaceae}

\section{Manilkara subsericea (Mart.) Dubard.}

Registration number: 195231, 195232 (V. Maia leg.)

Distribution: Brazil (Minas Gerais, Espírito Santo, Rio de Janeiro, São Paulo, Paraná, Santa Catarina).

Points of occurrence: RBM: MLP - 400, 1400, 2200, 2800, 3200, 4800, 5600, 6200m; ZBP - 200-600, 1400-4400, 4800, 5400-6200m. RI: 200-1400, 1800-2200, 2800, 3200-3400, 3800-4000m. RC: CLP - 200, 1200-1600, 2000, 2400m; PGP - 400-600, 1400-2600, 3000m.

Gall (Fig. 88). Circular leaf gall; length: 0.7-0.4 cm; width: 0.4-0.2 cm; glabrous; monothalamous; green. Gall maker: Cecidomyiidi. Pupation in the gall. Number of gall midge larvae/gall: 1. Localities: RBM, RI and RC. Material: RBM: 2 larvae (25.I.1998), 8 galls (26.XI.1997); RI: 1 larva (04.IX.1998), 9 pupal exuviae (2 on 10.VII.1998, 7 on 02.X.1998), 1 male (10.VII.1998), 2 galls (03.IV.1998); RC: 2 exuviae (28.XI.1998), 16 galls (23.V.1998). Other arthropods: inquiline Contarinia sp. (Cecidomyiidae): 1 male (24.X.1987); parasitoids - Clesterocerus sp. and Hadrotichodes sp. (Hymenoptera: Eulophidae). Periods of gall occurrence: March-January. Points of gall occurrence: RBM: MLP - 1400, 2800, 5600, 6200m; ZBP- 400-600, 1400-1600, 2200, 2600, 3000-3200m; RI: 400, 800, 1000,1400, $1800,2000,2800,3200-3400,3800-4000 \mathrm{~m}$; RC: CLP - 200, 1200-1600, 2400, 2600m; PGP - 400-600, 1600-1800, 2200, 2600m. Comments. Gall described by GAGNÉ (1994).

Gall (Fig. 89). Ovoid bud gall with a spine-like projection at the apex; length: $0.7 \mathrm{~cm}$; width: $0.3 \mathrm{~cm}$; glabrous; bi- or monothalamous; green. Gall maker: Cecidomyiidi (Cecidomyiidae). Pupation in the gall. Number of gall midge larva/chamber: 1. Locality: RC. Material: 9 larvae (1 on 29.VI.1998, 2 on 29.VIII.1998, 2 on 25.IX.1998, 1 on 31.X.1998, 1 on 17.III.1999), 5 pupal exuviae (3 col.29.VIII.1998, emerg.: 30.VIII.1998; 2 col. 31.X.1998, emerg.: 04.XI.1998), 6 males (3 col. 29.VIII.1998, emerg.: 30.VIII.1998; 1 col. 25.IX.1998, emerg.: 04.X.1998, 1 col. 31.X.1998, emerg.: 04.XI.1998, 1 col. 27.II.1999, emerg.: 27.II. 1999), 1 female (col. 31.X.1998, emerg.: 04.XI.1998).Other arthropods: parasitoid - Torymidae 
(Hymenoptera). Periods of gall occurrence: May-June, September-November, January-February. Points of gall occurrence: RC: CLP - 200, 1400, 2000, 2400m; PGP - 400-600, 1400-1600m.

Gall (Fig. 90). Tubular leaf gall; length: $0.2 \mathrm{~cm}$; width: $0.1 \mathrm{~cm}$; glabrous; monothalamous; yellow. Number of galls/leaf: 1-17. Gall maker: Asphondyliini, near to Macroporpa Rübsaamen, 1916 (Cecidomyiidae). Pupation in the gall. Number of gall midge larvae/gall: 1. Locality: RC. Material: 13 larvae (12 on 29.I.1999, 1 on 17.III.1999); 7 pupal exuviae (3 col.: 31.X.1998, emerg.: 04.XI. 1998-05.XI.1998; 2 on 27.XI.1998, 1 on 28.XI.1998, 1 col.: 29.I.1999, emerg.: 09.II.1999), 3 males (col. 28.XI.1998, emerg.: 30.XI.1998). Other arthropods: parasitoids - Eurytomidae and Platygastridae(Hymenoptera). Periods of gall occurrence: June-December, February-March. Points of gall occurrence: the same ones recorded to the host plant.

\section{Pouteria caimito (R. \& P.) Radlk. var. laurifolia (Gomes) Baehni}

Registration number: 161037 (A. Souza leg.)

Distribution: Brazil, Colombia, Peru and Trinidad.

Points of occurrence: RBM: MLP - 3000, 3600, 4000, 5800m; ZBP 200-400, 800, 3200, 6200m.

Gall (Fig. 91). Tubular leaf gall; length: 0.5-0.3 cm; width: 0.1-0.2 cm; glabrous; monothalamous; green. Number of galls/leaf: 1-7. Gall maker: Cecidomyiidi. Pupation in the gall. Number of gall midge larvae/gall: 1. Localities: RBM and RI. Material: RBM: 21 larvae (5 on 02.XII.1989, 4 on 05.VII.1995, 4 on 06.XII. 1997, 8 on 05.IV.1998), 3 males (2 on 23.III.1987, 1 on 06.XII.1997). Other arthropods: inquiline - Trotteria sp. (Cecidomyiidae): RBM: 2 pupal exuviae ( 1 on 06.XII.1997, 1 on 16.V.1998), 5 female (3 on 27.IV.1987, 1 on 21.V.1988, 1 on 13.VIII.1998), 5 males (4 on 27.IV.1987, 1 on 21.V.1988); parasitoids (Hymenoptera) - Eupelmidae, Platygastridae and Dimeromicrus cecidomyiae. Periods of gall occurrence: March-May, July, October-December. Points of gall occurrence: RBM: MLP - 3000, 3600m; ZBP - 200, 800, 3200m; RI: 4000m.

\section{Pouteria venosa (Mart.) Baehni (= Pouteria marginata)}

Registration number: 145923 (José Augusto leg.)

Distribution: Brazil (Amazonas, Bahia, Minas Gerais, Rio de Janeiro, São Paulo, Paraná, Santa Catarina).

Points of occurrence: RBM: MLP - 200, 800, 1200, 4000-6600m; ZBP $2200,2600-4000,4400-4800,5200-5400,5800,6200-6600 m$. RI: $1200,1800-$ $2200,3200 \mathrm{~m}$.

Figs 88-100. Restinga galls or damage. (88-92) on Sapotaceae: (88-90) on Manilkara subsericea (Sapotaceae): (88) leaf gall; (89) bud gall; (90) tubular leaf gall; (91) tubular leaf gall on Pouteria caimito var. laurifolia; (92) leaf gall on Pouteria venosa; (93) leaf gall on Smilax rufescens (Smilacaceae); (94-95) on Smilax rufescens (Smilacaceae): (94) larvae in fruits; (95) midvein swelling; (96-99) on Solanaceae - (96-97) on Aureliana fasciculata: (96) rolled young leaf; (97) elliptical leaf gall; (98) globular leaf gall on Solanum affine; (99) leaf blister on Solanum inaequale; (100) globular gall on Lantana camara (Verbenaceae). 

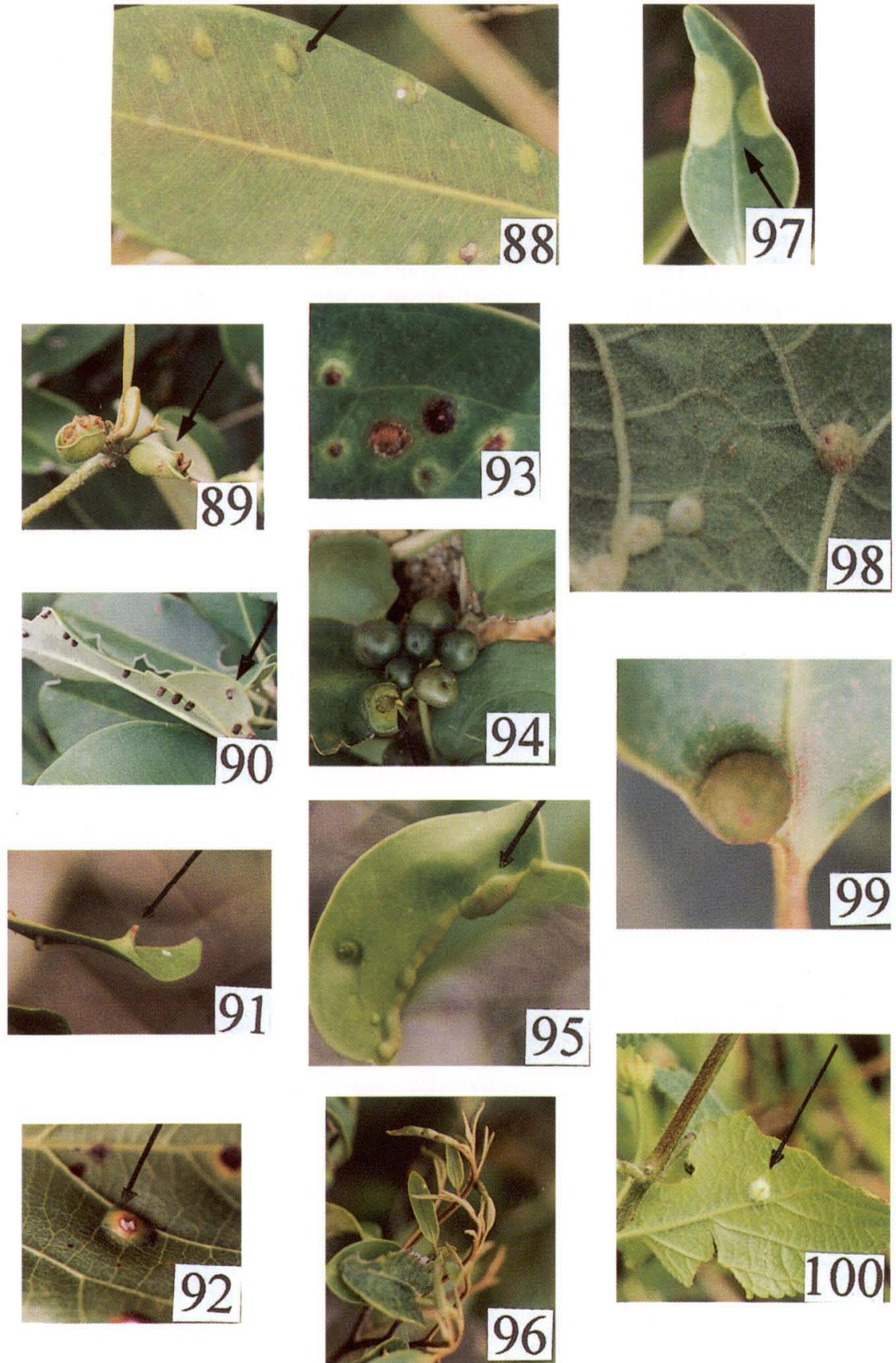

Revta bras. Zool. 18 (2): 583 - 629, 2001 
Gall (Fig. 92). Circular leaf gall; diameter: 0.4-0.5 cm; glabrous; monothalamous; green. Number of galls/leaf: 1-21. Gall maker: Lopesiini (Cecidomyiidae). Pupation in the gall. Number of gall midge larvae/gall: 1. Localities: RBM and RI. Material: RBM: 15 larvae (10 on 02.XII.1989, 3 on 16.XII.1989, 1 on 05.VI.1998, 1 on 03.VII.1998), 4 pupal exuviae (3 on 17.IV.1998, 1 on 08.IX.1998), 12 females (4 on 22.I.1987, 4 on 27.IV.1987, 1 on 22.IX.1987, 1 on 21.V.1988, 1 on VI.1988, 1 on 13.VIII.1988), 18 males (12 on 22.I.1987, 1 on 23.III.1987, 2 on 27.IV.1987, 1 on 22.IX.1987, 1 on 21.V.1988, 1 on 06.XII.1997), 11 galls (25.I.1998); RI: 16 pupal exuviae ( 9 on 10.VII.1998, 7 on 04.IX.1998), 12 females (6 on 10.VII.1998, 6 on 04.IX.1998), 3 males (10.VII.1998). Other arthropods: parasitoids (Hymenoptera) - Eupelmidae, Torymidae, Tetrastichinae, n. gen (Eulophidae) and Eurytomidae. Periods of gall occurrence: January, March-June, August-September, December. Points of gall occurrence: RBM: MLP - 200, 4600, 6000, 6400m; ZBP - the same ones recorded to the host plant; RI: 1200, 2000m.

\section{Smilacaceae}

\section{Smilax rufescens Griseb}

Registration number: 195254, 195255 (V. Maia leg.)

Distribution: Brazil (Amazonas, Rondônia, Maranhão, Alagoas, Bahia, Espírito Santo, Rio de Janeiro, São Paulo, Paraná, Santa Catarina).

Points of occurrence: RBM: MLP - 200-400, 1000, 1400-3800, 4400, 5000, 5400-5600, 6200m; ZBP - 200, 600-1600, 2200-2400, 3000, 3400, 4000, 4400m. RC: CLP- 200-800, 1200-4200m; PGP - 200-400, 800-3400m.

Gall (Fig. 93). Circular leaf gall; diameter: $0.3 \mathrm{~cm}$; glabrous; monothalamous; green or yellow. Number of galls/leaf: 3-78. Gall maker: Smilasioptera candelariae Möhn, 1975 (Cecidomyiidae). Pupation in the gall. Number of gall midge larvae/gall: 1. Localities: RBM, RI and RC. Material: RBM: 5 larvae (1on 07.VII.1987, 1 on 15.XI.1989, 3 on 06.XII.1997), 15 pupae (12 on 05.VI.1992, 3 on 06.XII.1997), 17 females (11 on 07.VII.1987, 5 on 09.IX.1987, 1 on 08.VII. 1992), 1 male (07.VII.1987), 25 galls (25.IV.1988); RC: 11 pupal exuviae (4 on 29.VI.1998, 7 on 31.X.1998), 7 females (2 on 29.VI.1998, 4 on 31.X.1998, 1 on 19.XII.1998), 7 males (31.X.1998), 35 galls (23.V.1998). Other arthropods: parasitoids - Pentastichus sp. (Hymenoptera: Eulophidae). Periods of gall occurrence: May-July, September-January. Points of gall occurrence: RBM: MLP - 200, 400, $1000 \mathrm{~m}$; ZBP- $1600 \mathrm{~m}$; RI: $3500 \mathrm{~m}$; RC: the same ones recorded to the host plant. Comments. Gall described by TAVARES (1909) and gall maker described by MÖHN (1975) on Smilax mexicanum.

Free living gall midge larva on immature fruits (diameter: $0.8 \mathrm{~cm}$ ) (Fig. 94). Number of gall midge larvae/fruit: 1-10. Gall midge: Clinodiplosis sp. Pupation In the gall. Locality: RBM. Material: 16 larvae (25.IV.1998). Periods of larva occurrence: April-may. Points of larva occurrence: $Z B P-600 \mathrm{~m}$.

Gall (Fig. 95). Midvein swelling; length: $0.7 \mathrm{~cm}$; width: $0.4 \mathrm{~cm}$; glabrous; monothalamous; green. Number of galls/leaf: 1-5. Gall maker: Cecidomyiinae. Pupation in the gall. Number of gall midge larvae/gall: 1. Localities: RBM and RC. 
Material: RC: 13 galls (8 on 29.IV.1998, 5 on 26.IX.1998). Periods of gall occurrence: November-February. Points of gall occurrence: RBM: MLP $-6200 \mathrm{~m}$; RC: CLP - 200, 1600m; PGP - 1400m.

\section{Solanaceae}

\section{Aureliana fasciculata (Vell.) Sendth.}

Registration number: 195260 (V. Maia leg.)

Distribution: Brazil (Acre, Bahia, Minas Gerais, Espírito Santo, Rio de Janeiro, São Paulo, Paraná, Santa Catarina, Rio Grande do Sul).

Points of occurrence: RC: CLP- 400-600, 1200m; PGP - 2600m.

Gall (Fig. 96). Rolled young leaf; length: $1.2 \mathrm{~cm}$; width: $0.2 \mathrm{~cm}$; glabrous; monothalamous; brownish. Gall maker: Cecidomyiinae. Pupation in the soil. Number of gall midge larvae/gall: 1. Locality: RC. Material: 2 larvae (28.VIII.1998), 3 galls (28.VIII.1998). Other arthropods: parasitoids - Hymenoptera. Periods of gall occurrence: August-September, October-February. Points of gall occurrence: the same ones recorded to the host plant.

Gall (Fig. 97). Elliptical leaf gall; length: $0.5 \mathrm{~cm}$; width: $0.3 \mathrm{~cm}$; glabrous; monothalamous; green. Gall maker: Cecidomyiinae. Pupation in the gall. Number of gall midge larvae/gall: 1. Locality: RC. Material: 1 larva (29.VIII.1998), 1 gall (29.VIII.1998). Periods of gall occurrence: August. Points of gall occurrence: PGP $-1200 \mathrm{~m}$.

\section{Solanum affine Sendth}

Registration number: 195261 (V. Maia leg.)

Distribution: Brazil (Minas Gerais, Rio de Janeiro, São Paulo, Paraná, Santa Catarina).

Points of occurrence: RBM: MLP - 200-800, 1600, 3600, 4000, 58006200m; ZBP - 600-800, 1400, 2200, 2800, 3400-3800, 4400-4800, 5600, 62006600m. RI: 1000-1200, 1600-2000, 2400, 2800-3000, 3400-4000m.

Gall (Fig. 98). Globular leaf gall; diameter: $0.4 \mathrm{~cm}$; glabrous; monothalamous; yellow. Gall maker: Cecidomyiinae. Pupation in the gall. Number of gall midge larvae/gall: 1. Localities: RBM and RI. Material: RBM: 27 larvae (23 on 24.X.1987, 4 on 10.X.1997); RI: 4 galls (12.VI.1998). Other arthropods: parasitoid - Miotropia sp. (Hymenoptera: Eulophidae). Periods of gall occurrence: June, October. Points of gall occurrence: RBM: MLP $-200 \mathrm{~m}$; RI: $1600 \mathrm{~m}$. Comments. Similar galls were described by TAVARES (1918) on Solanum sp.

\section{Solanum inaequale Vell.}

Registration number: 195597 (V. Maia leg.)

Distribution: Brazil (Ceará, Minas Gerais, Espírito Santo, Rio de Janeiro, São Paulo, Paraná, Santa Catarina, Rio Grande do Sul).

Points of occurrence: RC: CLP - 2000m.

Gall (Fig. 99). Leaf blister; length: $0.5 \mathrm{~cm}$; width: $0.3 \mathrm{~cm}$; glabrous; monothalamous; green. Gall maker: Cecidomyiinae. Pupation in the gall. Number of gall 
midge larvae/gall: 1. Locality: RC. Material: RBM: 1 larva (28.XI.1998), 4 pupal exuviae (3 on 28.XI.1998, 1 on 21.II.1999). Other arthropods: parasitoid - Hymenoptera. Periods of gall occurrence: November, February. Points of gall occurrence: CLP $-2000 \mathrm{~m}$.

\section{Verbenaceae}

\section{Lantana camara L.}

Registration number: 195267, 195268 (V. Maia leg.)

Distribution: Tropical and subtropical Americas. Few specimens in Asia and Africa. In Brazil, it occurs in Minas Gerais, Rio de Janeiro, São Paulo, Paraná.

Points of occurrence: RBM: MLP - 200, 400m.

Gall (Fig. 100). Globular leaf gall; diameter: $0.4 \mathrm{~cm}$; hairy; monothalamous; green. Number of galls/leaf: 1-5. Gall maker: Schismatodiplosis lantanae Rübsaamen, 1907 (Cecidomyiidae). Pupation in the gall. Number of gall midge larvae/gall: 1. Locality: RBM. Material: 3 pupal exuviae (03.VII.1998), 1 female (05.VI.1992), 5 males (1 on 05.VI.1998, 4 on 03.VII.1998), 7 galls (5 on 05.VI.1998 2 on 03.VII.1998). Periods of gall occurrence: June-July. Points of gall occurrence: MLP - 200, 400m. Comments. Gall and gall maker described by RÜBSAAMEN (1907); gall illustration showed by HOUARD (1933).

\section{New records}

New records of localities and arthropods in galls and new associations between gall midges and host plant.

Localities: Asphondylia cordiae Möhn, 1959 in Brazil (previous register: El Salvador); Alycaulus Rübsaamen, 1916 in State of Rio de Janeiro (previous registers: Amazonas, Brazil; Colombia; Costa Rica and El Salvador); Camptoneuromyia Felt, 1908 in Brazil (previous registers: El Salvador, Colombia, São Vicente and Trinidad) and Proasphondylia guapira Maia, 1993 in Maricá city (previous register: Arraial do Cabo city).

Association gall midges X host plant: Clinodiplosis Kieffer, 1895 on Burseraceae, Malpighiaceae and Smilacaceae; Dasineura Rondani, 1840 on Chrysobalanaceae; Contarinia Rondani, 1860 on Ochnaceae and Sapotaceae; Asphondylia cordiae on Cordia verbenacea (previous registers: on Cordia alba and Cordia dentata) and Schizomyia Kieffer, 1889 on Malpighiaceae.

\section{CONCLUSIONS}

Cecidomyiinae galls are very abundant in restinga environments, where they are associated with many plant families. Among them, the Myrtaceae shows the greatest richness of galls. This family as well as Leguminosae and Asteraceae are well represented in restingas, but only the first is rich in galls, result that differs from the South and Central Americas pattern suggested by HOUARD (1933).

The majority of the restinga galls occurs on the leaves, confirming a world trend as showed by MANI (1964).

The Cecidomyiidae genera best represented in this environment are: Clinodiplosis, Asphondylia, Stephomyia, Dasineura and Neolasioptera. 
The geographical distribution of four species of gall midge were enlarged and new records of host plant families were made.

Concerning the arthropod fauna associated with galls, the parasitoids (Hymenoptera), especially Eulophidae and Eurytomidae are the most common secondary dwellers in galls, suggesting that these families are the most important natural enemies of Cecidomyiinae in restingas.

Sorensen index showed that the Cecidomyiidae fauna of RBM and RI are more similar than that of $\mathrm{RC}$, what indicates a positive relation between geographical proximity and fauna similarity.

ACKNOWLEDGEMENTS. I wish to thank the botanists of Museu Nacional/UFRJ, specially MsC Andréa Costa, Dr. Rui Alves, Dr. Roberto L. Esteves, Ricardo L. de Moura and MsC Ivete Maria da Silva, as well as Dr. Graziela M. Barroso (Jardim Botânico, Rio de Janeiro) and Dr. José R. Pirani (Universidade de São Paulo) for host plants identification. Also Dr. John La Salle (International Institute of Entomology, Londres, England), Dr. Antônio Mayhé Nunes (Universidade federal Rural do Rio de Janeiro) and Dr. Mark Harvey (Western Australia Museum, Austrália) for micro-hymenoptera, Formicidae and pseudoscorpiones identification respectively. I am grateful to Dr. GAGNÉ (Smithsonian Institute, USDA, USA) and to Dr. Márcia Souto Couri for the manuscript review; to CAPES ("Fundação Coordenação de Aperfeiçoamento de Pessoal de Nivel Superior") and FAPERJ ("Fundação de Amparo à Pesquisa do Estado do Rio de Janeiro" - Proc. E-26/151.714/1999) for financial support from 1996 until 1999 and from 2000, respectively, and to Raul G. de Araújo Neto for the galls photographies.

\section{REFERENCES}

Araújo, D.S.D.; A. CostA; A.S. DE OliveirA \& R.L. DE MourA. (in press). Listagem das espécies vasculares da Restinga de carapebus e Arredores, R.J. Publ. Avulsas Museu Nacional.

AraúJo, D.S.D. \& R.P.B. HenriQues. 1984. Análise florística das restingas do Estado do Rio de Janeiro, p. 195-216. In: L.D. DE LACERDA; R. CERQUEIRA \& B. TURCQ (Eds). Restingas: origem, estrutura e processos. Niterói, CEUFF, Univ. Federal Fluminense, 475p.

araújo, D.S.D.; F.R. Scarano; C.F.C. de SÁ; B.C. Kurtz; H.L.T. Zaluar; R.C.M. Montezuma \& R.C. DE OliveIRA. 1998. Comunidades Vegetais do Parque Nacional da Restinga de Jurubatiba, p. 39-62. In: A.F. Esteves (Ed.). Ecologia das lagoas costeiras do Parque Nacional da Restinga de Jurubatiba e do município de Macaé (RJ). Rio de Janeiro, Univ. Federal do Rio de Janeiro, 464p. Barroso, G.M. \& M. Do C.M. MARques. 1997. Myrtaceae, p. 314-382. In: M. Do M. C.MARques; A.S. DA VAZ \& R. MARquete (Eds). Flórula da APA Cairuçu, Parati, R.J. Espécies vasculares. Rio de Janeiro, Série Estudos e Contribuições, JBRJ, Vol. 14, 576p.

Courı, M.S. \& V.C. MalA. 1992. Considerações sobre Pisphondylia Möhn, 1960 (Diptera, Cecidomyiidae, Asphondyliidi), com descrição de uma espécie nova do Brazil. Revta bras. Ent. 36 (4): 729-730.

Cronquist, A. 1988. The Evolution and Classification of Flowering Plants. New York, New York Botanical Garden, 535p.

FERnANDES, G.W.; E. TAMEIRÃO-Neto \& R.P. Martins. 1988. Ocorrência e caracterização de galhas entomógenas na vegetação do campus Pampulha da Universidade Federal de Minas Gerais. Revta bras. Zool. 5 (1): 11-29.

GaGné, R.J. 1981. Cecidomyiidae: 257-292. In: McAlpine, J.F.; B.V. Peterson; G.E. Shewell; H.J. Teskey; J.R. Vockeroth \& D.M. Wood (Eds). Manual of Neartic Diptera. Ottawa, Research Branch Agriculture Canada, Vol. 1, 674p.

- 1989. The Plant-Feeding Gall Midges of North America. Ithaca, Cornell Univ. Press, 
$356 \mathrm{p}$.

. 1994. The Gall Midges of the Neotropical Region. Ithaca, Cornell Univ. Press, 352p.

Houard, C. 1933. Les Zoocécidies des Plantes de l'Amérique du Sud et de l'Amérique Central. Paris, Hermann \& Cie, 549p.

MAIA, V.C. 1993a. Descrição de duas espécies novas de Cecidomyiidae (Diptera) associadas a Eugenia spp. (Myrtaceae). Revta bras. Zool. 37 (4): 717-721.

-1993b. Considerações sobre Proasphondylia Felt (Diptera, Cecidomyiidae) com descrições de duas espécies novas associadas com Guapira opposita (Velloso) Reitz (Nyctaginaceae). Revta bras. Zool. 10 (2): 215-218.

. 1993c. Considerações sobre Stephomyia Tavares (Diptera, Cecidomyiidae, Asphondyliidi) com descrição de quatro espécies novas associadas com Eugenia L. e Neomitranthes obscura (DC.) Legr. (Myrtaceae). Revta bras. Zool. 10 (3): 521-530.

.1994. Myrciarianyia bivalva, gen. n. e sp. n. (Diptera, Cecidomyiidae, Oligotrophini) associado com Myrciaria floribunda (Camb.) Legr. (Myrtaceae) no Brazil. Revta bras. Zool. 11 (4): 635-639.

- 1995a. Dois gêneros novos de Cecidomyiidae (Diptera) associados a Myrtaceae na Restinga da Barra de Maricá, Rio de Janeiro, Brazil. Revta bras. Zool. 12 (3): 567-574.

- 1995b. Três espécies novas de Dasineura Rondani (Diptera, Cecidomyiidae) associadas a Myrtaceae na Restinga da Barra de Maricá, Rio de Janeiro. Revta bras. Zool. 12 (4): 1001-1008.

- 1996a. Cordiamyia globosa, gen.n. e sp.n. 1840 (Diptera, Cecidomyiidae, Cecidomyiidi) associada com Cordia verbenacea DC. (Boraginaceae) no Brazil. Revta bras. Zool. 13 (3): 579-583. . 1996b. Clusiamyia nitida, gen.n. e sp.n. (Diptera, Cecidomyiidae, Cecidomyiidi) associada com Clusia lanceolata Camb. (Clusiaceae) no Brazil. Revta bras. Zool. 13 (4): 829-832.

-1999. Artrópodes associados às galhas de cecidomyiidae (Diptera) em Eugenia rotundifolia (Myrtaceae) e Clusia lanceolata (Clusiaceae) em uma restinga do Rio de Janeiro, Brasil. Iheringia Sér. Zool., 87: 75-79.

MaiA, V.C.; M.S. Couri \& R.F. Monteiro. 1992. Sobre seis espécies de Asphondylia Loew, 1850 do Brazil (Diptera, Cecidomyiidae). Revta bras. Ent. 36 (3): 653-661.

MaIA, V.C. \& M.S. Couri. 1993. Descrição de três espécies novas de Bruggmannia Tavares, 1906 cecidógenas (Diptera: Cecidomyiidae, Asphondyliidi) do BRAZIL associadas com Guapira opposita (Nyctaginaceae). Rev. Brasil. Biol. 53 (2): 209-215.

MAiA, V.C. \& R.F. Monteiro. 1999. Espécies cecidógenas (Diptera, Cecidomyiidae) e parasitóides (Hymenoptera) associadas a Guapira opposita (Vell.) Reitz (Nyctaginaceae) na Restinga da Barra de Maricá, Rio de Janeiro. Revta bras. Zool. 16 (2): 483-487.

MANI, M.S. 1964. Ecology of Plant Gall. The Hague, Junk, 434p.

Mariz, G. 1974. Chaves para as espécies de Clusia nativas do Brazil. Mem. Inst. Bioc. Univ. Fed. Pernambuco 1: 249-314.

MöHN, E. 1959. Gallmücken (Diptera, Itonididae) aus El Salvador. 1. Teil. Senck. Biol. 40: 297-368. . 1961. Gallmücken (Diptera, Itonididae) aus El Salvador. 4. Zur Phylogenie der Asphondyliidi der neotropischen und holarktischen Region. Senck. Biol. 42: 131-330.

- 1964. Gallmücken (Diptera, Itonididae) aus El Salvador. 6. Teil. Lasiopteridi. Deut. Entomol. Zeit 11: 47-143.

- 1975. Gallmücken (Diptera, Itonididae) aus El Salvador. 8. Teil. Lasiopteridi. Sttutgarter Beitr. Naturk (A) 276: 1-101.

Monteiro, R.F.; F. FerraZ; V.C. MAI^ \& M.A.P. Azevedo. 1993. Galhas entomógenas em restingas: uma abordagem preliminar. III Simpósio de Ecossistemas da Costa Brasileira, vol. 3, ACIESP 87 , p. $210-220$.

MoreirA, B.A. \& C.M. Rizzini. 1997. Loranthaceae e Viscaceae da APA de Maricá, Rio de Janeiro, Brazil. Acta Botanica BraSilica 11 (1): 1-8.

PranCE, G.T. 1972. Chrysobalanaceae. Flora Neotropica. New York, Hafner Press, Monogr. 9, 410p. ReITZ, P.R. 1968. Sapotaceae, 1-72. In: P.R. ReITz. Flórula Ilustrada Catarinense. Itajai, Livraria 
Blumenauense S.A., 72p.

Rizzini, C.T. 1979. Tratado de Fitogeografia do Brasil. São Paulo.Hucitec, Vol. 2, 54p.

RÜBSAAMEN, E.H. 1905. Beiträge zur Kenntnis aussereuropäischer Zoocecidien. II. Beitrag: Gallen aus Brazilien und Peru. Marcellia 4: 65-85, 115-138.

- 1907. Beiträge zur Kenntnis aussereuropäischer Zoocecidien. III. Beitrag: Gallen aus Brazilien und Peru. Marcellia 6: 110-173.

1916. Beiträge zur Kenntnis aussereuropäischer Zoocecidien. III. Beitrag: Gallen aus Brazilien und Peru. Sitzungsberitche der Gesellschaft Naturk. Freunde zur Berlin 1915: 431-481.

Silva, J.G. DA \& A.S. DE Oliveira. 1989. A Vegetação de Restinga do Município de Maricá, RJ. Acta bot. bras. 3 (2): 253-272.

Silva, J.G. DA \& G.V. SOMNER. 1984. A Vegetação da Restinga na Barra de Maricá, RJ, p. 217-224. In:

L.D. DE LACERda; R. Cerqueira \& B. TURCQ (Eds). Restingas: Origem, Estrutura e Processos. Niterói, CEUFF, Univ. Federal Fluminense, 475p.

TAVARES, J.S. 1909. Contributio prima ad cognitionem cecidologiae Braziliae. Brotéria, Sér. Zool. 5: $5-28$.

1918. Cecidologia Brazileira. Cecídias que se criam nas plantas das familias das Verbenaceae, Euphorbiaceae, Malvaceae, Anacardiaceae, Labiatae, Rosaceae, Anonaceae, Ampelidaceae, Bignoniaceae, Aristolochiaceae e Solanaceae. Brotéria, Sér. Zool., 16: 21-68. - 1920. Cecidologia Brazileira. Cecídias que se criam nas plantas das famílias das Leguminosae, Sapotaceae, Lauraceae, Myrtaceae, Punicaceae, Aurantiaceae, Malpighiaceae, Sapindaceae, Umbelliferae, Loranthaceae, Apocynaceae, Urticaceae, Salicaceae e Graminea. Brotéria, Sér. Zool., 18: 82-125. 1922. Cecidologia Brazileira. As restantes famílias. Brotéria, Sér. Zool., 20: 5-48.

1925. Nova contribuição para o conhecimento da Cecidologia Brazileira. Brotéria, Sér.

Zool., 22: 5-55.

Recebido em 23.III.2000; aceito em 08.VI.2001. 